\title{
Field quantization in dielectric media and the generalized multipolar Hamiltonian
}

\author{
B. J. Dalton, ${ }^{1,2}$ E. S. Guerra, ${ }^{1}$ and P. L. Knight ${ }^{1}$ \\ ${ }^{1}$ Optics Section, Blackett Laboratory, Imperial College of Science, Technology and Medicine, London SW7 2BZ, United Kingdom \\ ${ }^{2}$ Physics Department, The University of Queensland, Brisbane, Queensland 4072, Australia
}

(Received 21 April 1995; revised manuscript received 14 February 1996)

\begin{abstract}
Canonical quantization of the electromagnetic (EM) field is carried out for the situation where the total charge and current densities are the sum of contributions from neutral dielectric atoms whose effect is to be described purely classically in terms of spatially dependent electric permittivity and magnetic permeability functions, and neutral, stationary radiative atoms whose interaction with the EM field is to be treated quantum mechanically. The coefficients for the expansion of the vector potential in terms of mode functions determined from a generalized Helmholtz equation are chosen as independent generalized coordinates for the EM field. The spatially dependent electric permittivity and magnetic permeability appear in a generalized Helmholtz's equation and the former also occurs in the mode function orthogonality and normalization conditions. The quantum Hamiltonian is derived in a generalized multipolar form rather than the minimal coupling form obtained in other work. The radiative energy is the sum of quantum harmonic oscillator terms, one for each mode. The modes are independent in the present case of exact mode functions associated with the spatially dependent electric permittivity and magnetic permeability, there being no direct mode-mode coupling terms. In the electric dipole approximation the electric interaction energy contribution for each mode and radiative atom is proportional to the scalar product of the dipole operator with the mode function evaluated at the atom, times the annihilation operator, plus the Hermitian adjoint. This form has been widely used in studies of radiative processes for atomic systems in dielectric media, and it is justified here via the canonical quantization procedure. The results apply to the theoretical treatment of numerous quantum optical experiments involving such interactions in the presence of passive, lossless, dispersionless, linear classical optics devices such as resonator cavities, lenses, beam splitters, and so on. An illustrative application of the theory for atomic decay in a one-dimensional Fabry-Perot cavity is given.
\end{abstract}

[S1050-2947(96)06707-8]

PACS number(s): 42.50.-p, 12.20.Ds, 32.80.-t

\section{INTRODUCTION}

Quantum optical experiments often involve the interaction of the electromagnetic (EM) field and radiative atoms in the presence of passive, lossless, dispersionless, linear classical optics devices such as resonator cavities, lenses, beam splitters, filters, and so on. In certain situations quantum treatments of the dielectric atoms in such "classical" devices together with the various reservoirs (for example, phonons associated with lattice vibrations) to which they may be coupled are required. For example, dispersion and absorption effects (connected by causality through Kramers-Krönig relations) may be important-such as when the quantum optical frequencies of interest are close to resonance frequencies for the dielectric atoms. Canonical quantization [1] of the system of EM field, radiative and dielectric atoms, and with the dielectric treated quantum mechanically as a harmonic polarization field has been carried out by Huttner and Barnett and by Ho and Kumar [2], and this procedure may be referred to as microscopic canonical quantization. However, in many cases it is possible to ignore quantum effects associated with the dielectric atoms (essentially the Langevin noise and associated dissipation) and to treat classical optics devices via spatially inhomogeneous linear electric permittivity $\varepsilon(\mathbf{R})$ and magnetic permeability $\mu(\mathbf{R})$ (if the material is magnetic) functions. Thus the basic idea in such an approach is to replace the dielectric atoms by the classically equivalent spatially dependent dielectric constant. To illustrate this, Fig.
1 shows a number of radiative atoms contained inside an optical cavity produced by two concave mirrors consisting of material made up of dielectric atoms and having a high permittivity $\varepsilon$. The cavity would be represented by a permittivity function $\varepsilon(\mathbf{R})$, which equals $\varepsilon$ inside the mirrors and $\varepsilon_{0}$ everywhere else. In recent years mirrors of very high reflectivity have been constructed using Bragg reflectors. Such

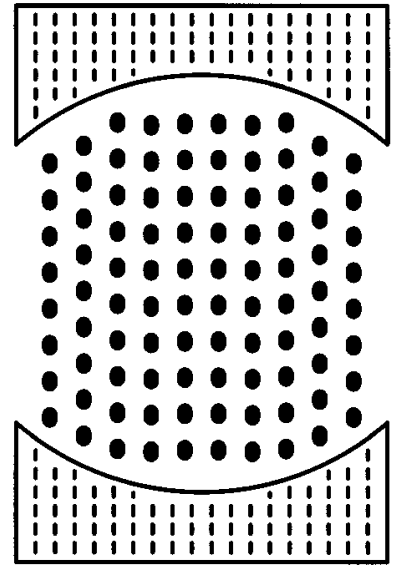

FIG. 1. Radiative atoms (shown as dots) are contained in an optical cavity between two mirrors (shown hatched) consisting of material made up of dielectric atoms and associated with a high electric permittivity $\varepsilon$. 
mirrors can be modeled in terms of a permittivity function $\varepsilon(\mathbf{R})$ with the required spatial periodicity.

In quantum optical experiments the primary interest is in the coupled radiative atoms and EM field system and in general the dynamic behavior of this system should be studied via a quantum treatment. The quantum Hamiltonian for the system is of fundamental importance and is derived in the present approach rather than merely assumed, with the dielectric atoms constituting the classical linear optical devices taken into account via appropriate spatially dependent electric permittivity functions. The radiative atoms and the EM field and their interaction are treated quantum mechanically. The quantum Hamiltonian for the radiation atoms and EM field is properly derived using the canonical quantization procedure [1]. This is based on a classical Lagrangian which takes the dielectric atoms into account via $\varepsilon(\mathbf{R}), \mu(\mathbf{R})$ and which gives the accepted classical Maxwell and Lorentz equations via the principle of least action (Lagrange's equations). This procedure may be referred to as macroscopic canonical quantization.

In carrying out the canonical quantization procedure, a number of steps are involved. First, the EM field is described via the vector potential $\mathbf{A}(\mathbf{R})$ and the scalar potential $\phi(\mathbf{R})$. However, the latter is then eliminated from the Lagrangian using a generalized radiation gauge that allows for the presence of the inhomogeneous electric permittivity $\varepsilon(\mathbf{R})$. The scalar potential is then just associated with Coulomb energy terms. Second, the original Lagrangian is replaced by a new Lagrangian which is designed to give a new Hamiltonian in the multipolar form $[1,3]$ rather than the minimal coupling form $[1,3]$. The new Lagrangian is still associated with the same action thereby leaving the Maxwell and Lorentz equations unchanged. Third, a set of independent, generalized coordinates to specify the EM field is obtained via the expansion of the vector potential in terms of vector mode functions. These are given as harmonic solutions for the vector potential with no radiative atoms present, and which satisfy a generalized Helmholtz equation taking the presence of the classical quantum optics device into account through terms depending on $\varepsilon(\mathbf{R})$. The spatially dependent electric permittivity is also involved in the mode function orthogonality and normalization conditions [4-6]. Fourth, the generalized momentum coordinates specifying the EM field are determined and the classical Hamiltonian in the multipolar form is constructed. Fifth, canonical quantization takes place by replacing the generalized coordinates and momenta (both for the EM field and for the radiative atom charged particles) by quantum operators satisfying standard equal time commutation laws. Sixth, the classical Hamiltonian is replaced by the quantum multipolar Hamiltonian and quantum field operators also replace the vector potential and its conjugate momentum field $\boldsymbol{\pi}(\mathbf{R})$. Commutation rules for the field operators $\hat{\mathbf{A}}(\mathbf{R})$ and $\hat{\boldsymbol{\pi}}(\mathbf{R})$ can then be determined, and these involve a generalization of the familiar transverse component of the delta function. The present macroscopic canonical quantization process will be restricted to the case where (i) the radiative atoms are neutral and stationary, and (ii) the dielectric medium is electrically neutral and nonconducting.

The quantum multipolar Hamiltonian resulting from the macroscopic canonical quantization procedure is a key result obtained here. Previous work on macroscopic canonical quantization involving the presence of radiative atoms and linear dielectrics $[4,5]$ has been carried out without the second step of forming a new Lagrangian and resulted in a quantum Hamiltonian in the minimal coupling form. Both the multipolar and minimal coupling Hamiltonians are exact and their complex forms contrast the simple Lagrangians on which they are based, indicating the impracticability of attempting to prejudge the Hamiltonian form on the basis of expected contributions to the energy of the system. This is not to say that the various terms in the Hamiltonians cannot be interpreted, and indeed a key result of the theory is the presence of radiation field energy terms which are equivalent to the Hamiltonian for a set of independent quantum harmonic oscillators, one for each mode of the field. This important result is the basis of the traditional photon picture for the quantum EM field which still applies even though the field is modified by the classical linear optics device. The modes are independent, there being no direct mode-mode coupling terms in the Hamiltonian for the present situation where exact vector mode functions ("true modes") for the dielectric system are used. Various studies that carry out macroscopic canonical quantization for the EM field in a linear dielectric medium with no radiative atoms present (see [7], for example) or which involve microscopic canonical quantization, where all charges are treated quantum mechanically (see [1,2], for example) also yield the photon picture. Work on macroscopic canonical quantization involving nonlinear dielectrics but with no radiative atoms present, has also been carried out, (see [8], for example), and also results in harmonic oscillator terms. Apart from the radiation field energy, the terms in the generalized multipolar Hamiltonian obtained here can be identified with the radiative particle kinetic energy, the radiative particle Coulomb energy, the polarization energy, the electric interaction energy, the magnetic interaction energy, and the diamagnetic energy. Leaving aside the last term the atom-field interaction leads to processes in which the usual one-photon absorptions or emissions are the basic step.

Approximate forms of the quantum multipolar Hamiltonian can be obtained based on an electric dipole approximation analogous to that used for the usual quantum multipolar Hamiltonian [1,3] based on quantization in free space, where $\varepsilon=\varepsilon_{0}$ everywhere. The electric interaction energy term is the sum of contributions for each mode and radiative atom. Each such contribution is the sum of a term plus its Hermitian adjoint, where the term is proportional to the scalar product of the atomic dipole operator and the mode function evaluated at the atom, times the annihilation operator multiplied by the electric field per photon. This form has been widely used [9] in studies of radiative processes for atomic systems in dielectric media, and in cavity quantum electrodynamics [10]. However, it is justified here using the canonical quantization procedure. The electric interaction energy can also be expressed as a sum of contributions for each atom of the scalar product of the atomic dipole operator with the conjugate momentum field operator evaluated at the atom, divided by the electric permittivity at the atom. In the present case of an inhomogeneous medium $\hat{\pi} / \varepsilon$ is not the same as the negative of the electric displacement operator, so the familiar expression for the electric interaction does not apply. 
Apart from the restrictions already indicated that the dielectric medium is nondispersive, nonabsorptive, and linear, the present macroscopic canonical quantization procedure is also not intended to apply to situations where inhomogeneous effects in the dielectric medium on an atomic scale are involved. Thus local field effects associated with crystal lattices lie outside the scope of the theory. Nevertheless, the results will be valid for a wide range of quantum optical situations, especially those associated with the rapidly developing area of cavity quantum electrodynamics [10].

The plan of this paper is as follows. In Sec. II the basic Lagrangian for radiative atoms and EM field in a nonhomogeneous linear dielectric and magnetic medium is introduced. In Sec. III a Lagrangian leading to a generalized multipolar Hamiltonian is obtained. In Sec. IV vector mode functions and generalized position and momentum coordinates for the field are examined, leading to further forms of the generalized multipolar Hamiltonian. Canonical quantization is carried out in Sec. V, leading to the generalized quantum multipolar Hamiltonian and its electric dipole approximation form. An illustrative example of the theory for atomic decay in a one-dimensional cavity is treated in Sec. VI and the main results are summarized in Sec. VII. Detailed derivations are placed in the Appendixes.

\section{BASIC LAGRANGIANS FOR RADIATIVE ATOMS AND EM FIELD IN NONHOMOGENEOUS LINEAR DIELECTRIC AND MAGNETIC MEDIUM. GENERALIZED RADIATION GAUGE}

\section{A. Macroscopic Lagrangian}

If $\mathbf{E}$ and $\mathbf{B}$ are the electric and magnetic fields, the Maxwell equations are

$$
\begin{gathered}
\boldsymbol{\nabla} \cdot \mathbf{B}=0, \\
\boldsymbol{\nabla} \times \mathbf{E}+\frac{\partial \mathbf{B}}{\partial t}=\mathbf{0}, \\
\boldsymbol{\nabla} \cdot \varepsilon_{0} \mathbf{E}=\rho, \\
\nabla \times \frac{\mathbf{B}}{\mu_{0}}-\varepsilon_{0} \frac{\partial \mathbf{E}}{\partial t}=\mathbf{j},
\end{gathered}
$$

where the charge and current densities $\rho$ and $\mathbf{j}$ are given by

$$
\begin{gathered}
\rho=\sum_{\xi \alpha} q_{\xi \alpha} \delta\left(\mathbf{R}-\mathbf{r}_{\xi \alpha}\right), \\
\mathbf{j}=\sum_{\xi \alpha} q_{\xi \alpha} \dot{\mathbf{r}}_{\xi \alpha} \delta\left(\mathbf{R}-\mathbf{r}_{\xi \alpha}\right) .
\end{gathered}
$$

Here $\xi=1,2, \ldots$ lists different atoms, and $\alpha=1,2, \ldots$ lists different particles within atom $\xi . q_{\xi \alpha}, M_{\xi \alpha}$ are the charge and mass for the $\xi \alpha$ particle at position $\mathbf{r}_{\xi \alpha}$. At present we consider all charges, whether associated with dielectric or magnetic media atoms or with radiative atoms. Associated with the charged particles we may define [1] a polarization density $\mathbf{P}$ and a magnetization density $\mathbf{M}$,

$$
\begin{aligned}
& \mathbf{P}=\sum_{\xi \alpha} q_{\xi \alpha} \int_{0}^{1} d u\left(\mathbf{r}_{\xi \alpha}-\mathbf{R}_{\xi}\right) \delta\left(\mathbf{R}-\mathbf{R}_{\xi}-u\left(\mathbf{r}_{\xi \alpha}-\mathbf{R}_{\xi}\right)\right), \\
& \mathbf{M}(R)= \sum_{\xi \alpha} q_{\xi \alpha} \int_{0}^{1} u d u\left(\mathbf{r}_{\xi \alpha}-\mathbf{R}_{\xi}\right) \\
& \times\left(\dot{\mathbf{r}}_{\xi \alpha}-\dot{\mathbf{R}}_{\xi}\right) \delta\left(\mathbf{R}-\mathbf{R}_{\xi}-u\left(\mathbf{r}_{\xi \alpha}-\mathbf{R}_{\xi}\right)\right) \\
&+\sum_{\xi \alpha} q_{\xi \alpha} \int_{0}^{1} d u\left(\mathbf{r}_{\xi \alpha}-\mathbf{R}_{\xi}\right) \\
& \times \dot{\mathbf{R}}_{\xi} \delta\left(\mathbf{R}-\mathbf{R}_{\xi}-u\left(\mathbf{r}_{\xi \alpha}-\mathbf{R}_{\xi}\right)\right)
\end{aligned}
$$

where for the $\xi$ atom $Q_{\xi}, M_{\xi}$, and $\mathbf{R}_{\xi}$ are the total charge, total mass, and center of mass position, respectively. Defining the free (or conduction) charge and current densities $\rho_{0}, \mathbf{j}_{0}$, corresponding to all charges being located at the center of masses $\mathbf{R}_{\xi}$ of the various atoms as

$$
\begin{gathered}
\rho_{0}(\mathbf{R})=\sum_{\xi} Q_{\xi} \delta\left(\mathbf{R}-\mathbf{R}_{\xi}\right), \\
\mathbf{j}_{0}(\mathbf{R})=\sum_{\xi} Q_{\xi} \dot{\mathbf{R}}_{\xi} \delta\left(\mathbf{R}-\mathbf{R}_{\xi}\right),
\end{gathered}
$$

then we can write [1]

$$
\begin{gathered}
\rho=\rho_{0}-\boldsymbol{\nabla} \cdot \mathbf{P}, \\
\mathbf{j}=\mathbf{j}_{0}+\frac{\partial \mathbf{P}}{\partial t}+\boldsymbol{\nabla} \times \mathbf{M},
\end{gathered}
$$

giving the charge density as the sum of the free charge density $\rho_{0}$ and the polarization charge density $-\boldsymbol{\nabla} \cdot \mathbf{P}$ and the current density as the sum of the free current density $\mathbf{j}_{0}$, the polarization current density $\dot{\mathbf{P}}$, and the magnetization current density $\boldsymbol{\nabla} \times \mathbf{M}$.

The replacement of dielectric media atoms via permittivity and permeability functions is based on writing the quantities $\rho, \mathbf{j}, \mathbf{P}, \mathbf{M}$ as the sum of contributions from charges (designated $D$ ) associated with the dielectric atoms and charges (designated $L$ ) associated with the radiative atoms,

$$
\begin{gathered}
\rho=\rho_{D}+\rho_{L}, \\
\mathbf{j}=\mathbf{j}_{D}+\mathbf{j}_{L}, \\
\mathbf{P}=\mathbf{P}_{D}+\mathbf{P}_{L}, \\
\mathbf{M}=\mathbf{M}_{D}+\mathbf{M}_{L} .
\end{gathered}
$$

A similar decomposition as in (6) applies to the constituents $\rho_{D}, \mathbf{j}_{D}$ or $\rho_{L}, \mathbf{j}_{L}$ making up the charge and current densities. For the case of neutral and nonconducting dielectric media we have

$$
\begin{aligned}
& \rho_{D 0}=0, \\
& \mathbf{j}_{D 0}=0,
\end{aligned}
$$


and using (6) for the case of the dielectric or magnetic media terms $\rho_{D}, \mathbf{j}_{D}$ the third and fourth Maxwell equations can be written as

$$
\begin{gathered}
\boldsymbol{\nabla} \cdot\left(\varepsilon_{0} \mathbf{E}+\mathbf{P}_{D}\right)=\rho_{L} \\
\boldsymbol{\nabla} \times\left(\frac{\mathbf{B}}{\mu_{0}}-\mathbf{M}_{D}\right)-\frac{\partial}{\partial t}\left(\varepsilon_{0} \mathbf{E}+\mathbf{P}_{D}\right)=\mathbf{j}_{L} .
\end{gathered}
$$

The electric displacement $\mathbf{D}$ and magnetic intensity $\mathbf{H}$ are related to the electric and magnetic fields $\mathbf{E}, \mathbf{B}$ and the polarization and magnetization densities $\mathbf{P}, \mathbf{M}$ as

$$
\begin{aligned}
& \mathbf{D}=\varepsilon_{0} \mathbf{E}+\mathbf{P}, \\
& \mathbf{H}=\frac{\mathbf{B}}{\mu_{0}}-\mathbf{M},
\end{aligned}
$$

so that

$$
\begin{gathered}
\mathbf{D}=\left(\varepsilon_{0} \mathbf{E}+\mathbf{P}_{D}\right)+\mathbf{P}_{L}, \\
\mathbf{H}=\left(\frac{\mathbf{B}}{\mu_{0}}-\mathbf{M}_{D}\right)-\mathbf{M}_{L} .
\end{gathered}
$$

It is these results that enable the inhomogeneous electric permittivity $\varepsilon(\mathbf{R})$ and magnetic permeability $\mu(\mathbf{R})$ associated with the linear dielectric or magnetic medium to be introduced. They are given via

$$
\begin{aligned}
& \varepsilon \mathbf{E}=\varepsilon_{0} \mathbf{E}+\mathbf{P}_{D}, \\
& \frac{\mathbf{B}}{\mu}=\frac{\mathbf{B}}{\mu_{0}}-\mathbf{M}_{D} .
\end{aligned}
$$

It is important to note that $\varepsilon, \mu$ are not the permittivity and permeability associated with all the charges, but only with the charges, currents $\left(\rho_{D}, \mathbf{j}_{D}\right)$ associated with the dielectric or magnetic medium. The distinction between the electric permittivity and magnetic permeability of the dielectric medium $\varepsilon, \mu$ and the total electric permittivity and magnetic permeability $\varepsilon_{T}, \mu_{T}$ for all the charges is an important one to make.

Maxwell's equations for electrically neutral, nonconducting dielectric and magnetic media, but with the radiative atom charges explicitly included, are obtained from (9) and (12) as

$$
\begin{gathered}
\boldsymbol{\nabla} \cdot \mathbf{B}=0, \\
\boldsymbol{\nabla} \times \mathbf{E}+\frac{\partial \mathbf{B}}{\partial t}=\mathbf{0}, \\
\boldsymbol{\nabla} \cdot \varepsilon \mathbf{E}=\rho_{L}, \\
\boldsymbol{\nabla} \times \frac{\mathbf{B}}{\mu}-\varepsilon \frac{\partial \mathbf{E}}{\partial t}=\mathbf{j}_{L}
\end{gathered}
$$

We thus have eliminated the dielectric or magnetic media charges which only appear via $\varepsilon(\mathbf{R}), \mu(\mathbf{R})$. The remaining charge and current densities are now only associated with the radiative atoms. The vector and scalar potentials $\mathbf{A}, \phi$ are related to the electric and magnetic fields $\mathbf{E , B}$ via the usual equations

$$
\begin{gathered}
\mathbf{E}=-\boldsymbol{\nabla} \phi-\frac{\partial \mathbf{A}}{\partial t}, \\
\mathbf{B}=\boldsymbol{\nabla} \times \mathbf{A} .
\end{gathered}
$$

A straightforward application of the principle of least action to the Lagrangian given by

$$
L=\sum_{\xi \alpha} \frac{1}{2} M_{\xi \alpha} \dot{\mathbf{r}}_{\xi \alpha}^{2}+\int d^{3} \mathbf{R} \mathcal{L},
$$

where

$$
\mathcal{L}=\frac{1}{2} \varepsilon \mathbf{E}^{2}-\frac{1}{2 \mu} \mathbf{B}^{2}+\mathbf{j}_{L} \cdot \mathbf{A}-\rho_{L} \boldsymbol{\phi}
$$

is the Lagrangian density, yields both the modified Maxwell's equations (13) and the Lorentz equations (17) for the classical motion of the charged particles associated with the radiative atoms.

$$
M_{\xi \alpha} \ddot{\mathbf{r}}_{\xi \alpha}=q_{\xi \alpha}\left(\mathbf{E}\left(\mathbf{r}_{\xi \alpha}\right)+\dot{\mathbf{r}}_{\xi \alpha} \times \mathbf{B}\left(\mathbf{r}_{\xi \alpha}\right)\right) .
$$

We may refer to the Lagrangian $L$ as the macroscopic Lagrangian, as the atoms in the dielectric or magnetic medium are now treated macroscopically via the electric permittivity and magnetic permeability. It is important to note that the Lagrangian (15) (and all subsequent expressions) only involves a sum $\xi \alpha$ over charges associated with the radiative atoms.

\section{B. Generalized radiation gauge and elimination of scalar potential}

From the third Maxwell equation (13) we find using (14)

$$
\boldsymbol{\nabla} \cdot(\varepsilon \boldsymbol{\nabla} \phi)+\boldsymbol{\nabla} \cdot\left(\varepsilon \frac{\partial \mathbf{A}}{\partial t}\right)=-\rho_{L} .
$$

In a generalized radiation (Coulomb) gauge we want the scalar potential $\phi$ to be determined from the charge density without any retardation effects. This can be accomplished via the use of the generalized radiation gauge [4,5]

$$
\boldsymbol{\nabla} \cdot \varepsilon \mathbf{A}=0
$$

In this case the scalar potential satisfies a generalized Poisson equation

$$
\nabla \cdot(\varepsilon \nabla \phi)=-\rho_{L} .
$$

This equation relates the scalar potential $\phi$ to the instantaneous positions $\mathbf{r}_{\xi \alpha}$ of all the radiative atom charges. This enables us to disregard $\phi$ as an independent field variable. Unlike the free space situation, however, the scalar potential will depend on the electric permittivity $\varepsilon(\mathbf{R})$ as well as on the position of the radiative charges. From the fourth Maxwell equation (13) we find using (14) that 


$$
\boldsymbol{\nabla} \times \frac{1}{\mu}(\boldsymbol{\nabla} \times \mathbf{A})+\varepsilon \frac{\partial^{2} \mathbf{A}}{\partial t^{2}}=\mathbf{j}_{L}-\varepsilon \boldsymbol{\nabla} \frac{\partial \phi}{\partial t},
$$

which is a generalized inhomogeneous wave equation for the vector potential $\mathbf{A}$. The term $\mathbf{j}_{L}-\varepsilon \boldsymbol{\nabla} \dot{\phi}$ acts as a source term.

The use of the generalized radiation gauge enables the scalar potential to be eliminated from the Lagrangian. Substituting for $\mathbf{E}$ via (14) in the term $\int d^{3} \mathbf{R}_{2}^{1} \varepsilon \mathbf{E}^{2}$ a straightforward vector calculus treatment making use of (19) and (20) gives $[4,5]$

$$
L=\sum_{\xi \alpha} \frac{1}{2} M_{\xi \alpha} \dot{\mathbf{r}}_{\xi \alpha}^{2}-V_{\mathrm{Coul}}+\int d^{3} \mathbf{R} \mathcal{L}_{C},
$$

where

$$
V_{\text {Coul }}=\frac{1}{2} \int d^{3} \mathbf{R} \phi \rho_{L}=\int d^{3} \mathbf{R} \frac{(\varepsilon \nabla \phi) \cdot(\varepsilon \nabla \phi)}{2 \varepsilon}
$$

and

$$
\mathcal{L}_{C}=\frac{1}{2} \varepsilon \dot{\mathbf{A}}^{2}-\frac{1}{2 \mu}(\boldsymbol{\nabla} \times \mathbf{A})^{2}+\mathbf{j}_{L} \cdot \mathbf{A}
$$

are the Coulomb energy and the radiative Lagrangian density. The Coulomb energy $V_{\text {Coul }}$ only depends on positions of radiative atom charges and the radiative Lagrangian density $\mathcal{L}_{C}$ only depends on the vector potential $\mathbf{A}$. It should be noted that the Coulomb energy (and hence the Lagrangian) only depends on the scalar potential $\phi$ in terms of the quantity $\varepsilon \nabla \phi$. It may appear that the vector potential $\mathbf{A}$ and the scalar potential via $\varepsilon \boldsymbol{\nabla} \phi$ are not determined uniquely by the generalized radiation gauge condition (19) and the generalized Poisson equation (20). In fact these conditions $d o$ give $\mathbf{A}$ and $\varepsilon \boldsymbol{\nabla} \phi$ uniquely, as is demonstrated in Appendix A.

\section{LAGRANGIAN AND GENERALIZED MULTIPOLAR HAMILTONIAN. CONJUGATE MOMENTUM FIELD}

The previous Lagrangian (22) can be used to calculate the conjugate momentum field, the minimal coupling Hamiltonian, and via a mode expansion and the canonical quantization procedure will result in a quantum generalized minimal coupling Hamiltonian obtained in previous work [4,5]. Here we wish to derive a quantum generalized multipolar Hamiltonian, so following the procedure used in standard treatments $[1,3]$ we add a term to the old Lagrangian (22) to produce a new Lagrangian that leads to a Hamiltonian in the multipolar form. The additional term is a total time derivative involving the vector potential $\mathbf{A}$ and the polarization density $\mathbf{P}_{L}$ associated with the radiative atoms. The action and hence the Maxwell or Lorentz equations are thus left unaltered. The new Lagrangian is

$$
L^{\prime}=L+\frac{d F}{d t},
$$

where

$$
F=-\int d^{3} \mathbf{R} \mathbf{P}_{L} \cdot \mathbf{A} .
$$

From now on we assume that the radiative atoms are electrically neutral, $Q_{\xi}=0$. Hence from (5)

$$
\begin{aligned}
& \rho_{L 0}=0, \\
& \mathbf{j}_{L 0}=0 .
\end{aligned}
$$

Using (27) and the second equation (6) $\mathbf{j}_{L}=\dot{\mathbf{P}}_{L}+\boldsymbol{\nabla} \times \mathbf{M}_{L}$ for the radiative charges it is easily seen that the new Lagrangian is given by

$$
\begin{aligned}
L^{\prime} & =\sum_{\xi \alpha} \frac{1}{2} M_{\xi \alpha} \dot{\mathbf{r}}_{\xi \alpha}^{2}-V_{\text {Coul }}+\int d^{3} \mathbf{R}\left\{\frac{1}{2} \varepsilon\left(\frac{\partial \mathbf{A}}{\partial t}\right)^{2}-\frac{1}{2 \mu}(\boldsymbol{\nabla} \times \mathbf{A})^{2}-\mathbf{P}_{L} \cdot\left(\frac{\partial \mathbf{A}}{\partial t}\right)+\mathbf{M}_{L} \cdot \nabla \times \mathbf{A}\right\} \\
& =\sum_{\xi \alpha} \frac{1}{2} M_{\xi \alpha} \dot{\mathbf{r}}_{\xi \alpha}^{2}-V_{\text {Coul }}+\int d^{3} \mathbf{R} \mathcal{L}_{C}^{\prime},
\end{aligned}
$$

where

$$
\mathcal{L}_{C}^{\prime}=\frac{1}{2} \varepsilon\left(\frac{\partial \mathbf{A}}{\partial t}\right)^{2}-\frac{1}{2 \mu}(\boldsymbol{\nabla} \times \mathbf{A})^{2}-\mathbf{P}_{L}^{\prime} \cdot\left(\frac{\partial \mathbf{A}}{\partial t}\right)+\mathbf{M}_{L} \cdot \boldsymbol{\nabla} \times \mathbf{A} .
$$

The new radiative Lagrangian density $\mathcal{L}_{C}^{\prime}$ involves a reduced polarization density $\mathbf{P}_{L}^{\prime}$, for which the explicit expression will be obtained later [see Eq. (53)]. This allows for $\mathbf{A}$ to be constrained via the generalized radiation gauge condition (19), so that for all such $\mathbf{A}$

$$
\int d^{3} \mathbf{R} \mathbf{P}_{L} \cdot \mathbf{A}=\int d^{3} \mathbf{R} \mathbf{P}_{L}^{\prime} \cdot \mathbf{A}
$$

In the usual free space treatment where $\varepsilon=\varepsilon_{0}$ everywhere and $\boldsymbol{\nabla} \cdot \mathbf{A}=0$ gives the usual radiation gauge condition, $\mathbf{P}_{L}^{\prime}$ would be the transverse component of $\mathbf{P}_{L}$. In the present situation although $\mathbf{P}_{L}^{\prime}$ turns out to be transverse, it is not the same as the transverse component of $\mathbf{P}_{L}$. This will be demonstrated later [see Eqs. (82) and (83)] by a particular example, but is not surprising in that whereas $\mathbf{P}_{L}^{\prime}$ must depend on $\varepsilon(\mathbf{R})$ in view of (19), $\mathbf{P}_{L}$ and its transverse component are independent of $\varepsilon(\mathbf{R})$.

From now on we also assume that the radiative atoms are 
stationary by assuming $M_{\xi} \longrightarrow \infty$. Thus

$$
\dot{\mathbf{R}}_{\xi}=\mathbf{0}
$$

and from (4) the magnetization density $\mathbf{M}_{L}$ is given as the simpler form

$$
\begin{aligned}
\mathbf{M}_{L}= & \sum_{\xi \alpha} q_{\xi \alpha} \int_{0}^{1} u d u\left(\mathbf{r}_{\xi \alpha}-\mathbf{R}_{\xi}\right) \\
& \times \dot{\mathbf{r}}_{\xi \alpha} \delta\left(\mathbf{R}-\mathbf{R}_{\xi}-u\left(\mathbf{r}_{\xi \alpha}-\mathbf{R}_{\xi}\right)\right) .
\end{aligned}
$$

The explicit dependence of the new Lagrangian (28) on the particle velocities $\dot{\mathbf{r}}_{\xi \alpha}$ and field velocity $\dot{\mathbf{A}}$ is

$$
\begin{aligned}
L^{\prime}= & \sum_{\xi \alpha} \frac{1}{2} M_{\xi \alpha} \dot{\mathbf{r}}_{\xi \alpha}^{2}-V_{\mathrm{Coul}}+\int d^{3} \mathbf{R}\left(\frac{1}{2} \varepsilon\left(\frac{\partial \mathbf{A}}{\partial t}\right)^{2}-\frac{1}{2 \mu}(\boldsymbol{\nabla} \times \mathbf{A})^{2}\right)+\sum_{\xi \alpha} \dot{\mathbf{r}}_{\xi \alpha} \int_{0}^{1} u d u q_{\xi \alpha} B\left(\mathbf{R}_{\xi}+u\left(\mathbf{r}_{\xi \alpha}-\mathbf{R}_{\xi}\right)\right) \times\left(\mathbf{r}_{\xi \alpha}-\mathbf{R}_{\xi}\right) \\
& -\int d^{3} \mathbf{R} \mathbf{P}_{L}^{\prime} \cdot\left(\frac{\partial \mathbf{A}}{\partial t}\right)
\end{aligned}
$$

The momenta $\mathbf{p}_{\alpha \xi}$ for the radiative atom charges and the conjugate momentum field $\boldsymbol{\pi}(\mathbf{R})$ for the EM field can be obtained [1] from the Lagrangian (33) and the radiative Lagrangian density (29) as

$$
\begin{gathered}
\mathbf{p}_{\xi \alpha}=M_{\xi \alpha} \dot{\mathbf{r}}_{\xi \alpha}+\int_{0}^{1} u d u q_{\xi \alpha} \mathbf{B}\left(\mathbf{R}_{\xi}+u\left(\mathbf{r}_{\xi \alpha}-\mathbf{R}_{\xi}\right)\right) \times\left(\mathbf{r}_{\xi \alpha}-\mathbf{R}_{\xi}\right), \\
\boldsymbol{\pi}=\varepsilon \frac{\partial \mathbf{A}}{\partial t}-\mathbf{P}_{L}^{\prime} .
\end{gathered}
$$

The new Hamiltonian can then be constructed [1] using the standard method as

$$
\begin{aligned}
H^{\prime}= & \sum_{\xi \alpha} \frac{\mathbf{p}_{\xi \alpha}^{2}}{2 M_{\xi \alpha}}+V_{\text {Coul }}+\int d^{3} \mathbf{R}\left(\frac{\left(\boldsymbol{\pi}+\mathbf{P}_{L}^{\prime}\right)^{2}}{2 \varepsilon}+\frac{(\boldsymbol{\nabla} \times \mathbf{A})^{2}}{2 \mu}\right)-\int d^{3} \mathbf{R}(\boldsymbol{\nabla} \times \mathbf{A}) \cdot \mathbf{M}_{L}^{\prime} \\
& +\sum_{\xi \alpha} \frac{q_{\xi \alpha}^{2}}{2 M_{\xi \alpha}}\left(\int_{0}^{1} u d u B\left(\mathbf{R}_{\xi}+u\left(\mathbf{r}_{\xi \alpha}-\mathbf{R}_{\xi}\right)\right) \times\left(\mathbf{r}_{\xi \alpha}-\mathbf{R}_{\xi}\right)\right)^{2},
\end{aligned}
$$

where the reduced magnetization density is given by

$$
\mathbf{M}_{L}^{\prime}=\sum_{\xi \alpha} q_{\xi \alpha} \int_{0}^{1} u d u\left(\mathbf{r}_{\xi \alpha}-\mathbf{R}_{\xi}\right) \times \frac{p_{\xi \alpha}}{M_{\xi \alpha}} \delta\left(R-\mathbf{R}_{\xi}-u\left(\mathbf{r}_{\xi \alpha}-\mathbf{R}_{\xi}\right)\right) .
$$

The details are given in Appendix B. This classical Hamiltonian, although restricted to the case of neutral, stationary radiative atoms, is in the multipolar form. However, the interpretation of the various terms is not yet clear and the Hamiltonian is not suitable as it stands for quantization, since we do not know the proper commutation rules for operators representing the vector potential and its conjugate momentum field and we also do not have an explicit expression for the reduced polarization density.

\section{MODE FUNCTIONS, GENERALIZED POSITION, AND MOMENTUM COORDINATES FOR THE EM FIELD, AND THE GENERALIZED MULTIPOLAR HAMILTONIAN}

\section{A. Basic idea for canonical quantization}

The possible approach of just replacing the fields $\mathbf{A}, \boldsymbol{\pi}$ by field operators $\hat{\mathbf{A}}, \hat{\boldsymbol{\pi}}$ in the Hamiltonian is an unsatisfactory quantization procedure. At this stage we would have to guesswhat the commutation rules for $\hat{\mathbf{A}}, \hat{\boldsymbol{\pi}}$ are; presumably they involve a generalization of the transverse $\delta$ function and include $\varepsilon(\mathbf{R})$ rather than $\varepsilon_{0}$. Also, $\mathbf{A}$ satisfies a constraint, the radiation gauge condition $\boldsymbol{\nabla} \cdot \varepsilon \mathbf{A}=0$, and this has to be taken into account in the quantization procedure. In a proper canonical quantization procedure the fields are expressed in terms of a suitable set of generalized independent coordinates, $q_{k}$ and the time derivative of the field will involve the time derivatives of the generalized coordinates $\dot{q}_{k}$, which play the role of generalized velocities. The Lagrangian is then expressed in terms of the $q_{k}, \dot{q}_{k}$ and the generalized momenta $p_{k}$ given via

$$
p_{k}=\frac{\partial L}{\partial \dot{q}_{k}^{*}}
$$

The complex conjugate allows for the case where the $q_{k}$ are complex. The Hamiltonian is then calculated via 


$$
H=\sum_{k}\left(\dot{q}_{k} p_{k}^{*}+\dot{q}_{k}^{*} p_{k}\right)-L
$$

where the sum is over independent $k$ and depends on the generalized coordinates $q_{k}$ and generalized momenta $p_{k}$.

Quantization then proceeds via the replacement of the generalized coordinates and momenta by quantum operators $q_{k} \rightarrow \hat{q}_{k}, p_{k} \rightarrow \hat{p}_{k}, q_{k}^{*} \rightarrow \hat{q}_{k}^{\dagger}, p_{k}^{*} \rightarrow \hat{p}_{k}^{\dagger}$, and which satisfy standard commutation rules, for which the nonzero results are

$$
\left[\hat{q}_{k}, \hat{p}_{k}^{\dagger}\right]=\left[\hat{q}_{k}^{\dagger}, \hat{p}_{l}\right]=i \hbar \delta_{k l} .
$$

Other physical quantities such as $\mathbf{A}, \boldsymbol{\pi}$, and $H$ can then also be replaced by quantum operators making use of their expressions in terms of the $q_{k}, p_{k}$ and their commutation rules derived from those applying to the $\hat{q}_{k}, \hat{p}_{k}$.

\section{B. Vector mode functions}

In our case we will introduce the required independent generalized coordinates via an expansion of the vector potential $\mathbf{A}$ in terms of the vector mode functions $\mathbf{A}_{k}$ applying for no radiative atoms present $\mathbf{j}_{L}=\rho_{L}=0$. This is the generalization of the usual expansion of $\mathbf{A}$ in terms of plane waves and the use of reciprocal space [1]. We need not restrict ourselves to the case of nonmagnetic media where $\mu=\mu_{0}$ throughout. For the case where $\mathbf{j}_{L}=\rho_{L}=0$ the generalized inhomogeneous wave equation (21) becomes a homogeneous wave equation and has harmonic solutions of the form

$$
\mathbf{A}=\mathbf{A}_{k}(\mathbf{R}) e^{i \omega_{k} t}
$$

where $\omega_{k}$ is the angular frequency. The vector mode functions $\mathbf{A}_{k}$ satisfy the generalized Helmholtz equation

$$
\boldsymbol{\nabla} \times \frac{1}{\mu}\left(\boldsymbol{\nabla} \times \mathbf{A}_{k}\right)=\omega_{k}^{2} \boldsymbol{\varepsilon} \mathbf{A}_{k} .
$$

It is easy to see from (42) that the vector mode functions satisfy the generalized radiation gauge condition (19),

$$
\boldsymbol{\nabla} \cdot \boldsymbol{\varepsilon} \mathbf{A}_{k}=0 \text {. }
$$

Such vector mode functions have been extensively used in other work involving the quantized EM field in dielectric media (see, for example, [4-6,11]) and the properties of the vector mode functions have been worked out, at least for the case where $\mu=\mu_{0}$ everywhere. The properties are obtained for the general case by demonstrating that the operator

$$
L=\frac{1}{\varepsilon} \nabla \times \frac{1}{\mu}(\nabla \times \cdots)
$$

is self adjoint in terms of integrals $\int d^{3} \mathbf{R} \varepsilon(\mathbf{R}) \ldots$, where $\varepsilon(\mathbf{R})$ acts as a weight function. From Eq. (42) the eigenvalues of $L$ are the $\omega_{k}^{2}$ and the $\mathbf{A}_{k}$ are the eigenfunctions. Details are given in Appendix C. The important properties of the vector mode functions are as follows: (i) the $\omega_{k}$ are all real (and positive); and (ii) the orthogonality and normalization conditions are given by

$$
\int d^{3} \mathbf{R} \varepsilon(\mathbf{R}) \mathbf{A}_{k}^{*}(\mathbf{R}) \cdot \mathbf{A}_{l}(\mathbf{R})=\delta_{k l} .
$$

This condition may be taken to apply to vector mode functions that are degenerate via the use of the Schmidt orthogonalization procedure. The index $k$ will be used to distinguish degenerate vector functions; these may, for example, have different polarization vectors $\boldsymbol{\varepsilon}_{k}$. Thus $k \rightarrow\left(\omega_{k}, i\right)$ where $i$ lists degenerate modes. Noting that the complex conjugate $\left(\mathbf{A}_{k}\right)^{*}$ satisfies the generalized Helmholtz equation with the same angular frequency $\omega_{k}$ as for the $k$ mode, we may define the $k^{*}$ mode via

$$
\left.\mathbf{A}_{k} * \mathbf{R}\right)=\left(\mathbf{A}_{k}(\mathbf{R})\right)^{*}
$$

This is degenerate with $\mathbf{A}_{k}$.

In the case of free space with $\varepsilon=\varepsilon_{0}$ everywhere the vector mode functions are the usual plane wave forms defined in quantization volume $V$,

$$
\mathbf{A}_{k}(\mathbf{R})=\frac{1}{\sqrt{\varepsilon_{0} V}} \boldsymbol{\varepsilon}_{k} e^{i \mathbf{k} \cdot \mathbf{R}}
$$

with the polarization vector $\boldsymbol{\varepsilon}_{k}$ and angular frequency $\omega_{k}$ satisfying

$$
\begin{gathered}
\mathbf{k} \cdot \boldsymbol{\varepsilon}_{k}=0, \\
\omega_{k}=c|\mathbf{k}|, \quad c=\frac{1}{\sqrt{\mu_{0} \varepsilon_{0}}} .
\end{gathered}
$$

In the free space case the $k^{*}$ mode would then have polarization vector $\left(\boldsymbol{\varepsilon}_{k}\right)^{*}$ and wave vector $-\mathbf{k}$.

\section{Expansion of vector potential}

The expansion of the vector potential in terms of the vector mode functions is written as

$$
\mathbf{A}=\sum_{k} q_{k} \mathbf{A}_{k}
$$

where the $q_{k}$ are the expansion coefficients. Using (45) we have

$$
q_{k}=\int d^{3} \mathbf{R} \varepsilon(\mathbf{R}) \mathbf{A}_{k}^{*}(\mathbf{R}) \cdot \mathbf{A}(\mathbf{R}) .
$$

Using the requirement that the vector potential is real we see using (46) that the expansion coefficients for the $k$ and $k^{*}$ modes are not independent, but are complex conjugates,

$$
q_{k} *\left(q_{k}\right)^{*} .
$$

In view of this and generalizing the free space case we then see that it is the set of $q_{k}$ defined in a suitable half space (in which only one of $q_{k}$ and $q_{k}$ *appear) that act as independent generalized coordinates describing the EM field. Thus we have

$$
A=\sum_{k}^{\prime}\left(q_{k} \mathbf{A}_{k}+q_{k}^{*} \mathbf{A}_{k}^{*}\right)
$$

where the sum $\Sigma_{k}^{\prime}$ is over the half space only. The set of $\dot{q}_{k}$ in the half space act as the generalized velocities. 


\section{Reduced polarization density}

Consider the expression

$$
\mathbf{P}_{L}^{\prime}(\mathbf{R})=\sum_{k} \varepsilon(\mathbf{R}) \mathbf{A}_{k}(\mathbf{R}) \int d^{3} \mathbf{R}^{\prime} \mathbf{P}_{L} \cdot \mathbf{A}_{k}^{*}
$$

Then with A given by the complex conjugate of the form (49) we have for arbitrary A satisfying the generalized radiation gauge condition (19)

$$
\begin{aligned}
\int d^{3} \mathbf{R} \mathbf{P}_{L}^{\prime} \cdot \mathbf{A}= & \sum_{k l} q_{l}^{*} \int d^{3} \mathbf{R} \mathbf{A}_{l}^{*}(\mathbf{R}) \cdot \varepsilon(\mathbf{R}) \mathbf{A}_{k}(\mathbf{R}) \\
& \times \int d^{3} \mathbf{R}^{\prime} \mathbf{P}_{L}\left(\mathbf{R}^{\prime}\right) \cdot \mathbf{A}_{k}^{*}\left(\mathbf{R}^{\prime}\right) \\
= & \sum_{k} q_{k}^{*} \int d^{3} \mathbf{R} \mathbf{P}_{L} \cdot \mathbf{A}_{k}^{*} .
\end{aligned}
$$

The orthogonality condition (45) has been used. But for the same arbitrary A

$$
\int d^{3} \mathbf{R} \mathbf{P}_{L} \cdot \mathbf{A}=\sum_{k} q_{k}^{*} \int d^{3} \mathbf{R} \mathbf{P}_{L} \cdot \mathbf{A}_{k}^{*}
$$

so that for all choices of the $q_{k}$ the condition (30) is satisfied with the reduced polarization density $\mathbf{P}_{L}^{\prime}$ given by (53). We may therefore take (53) as giving the expression for the reduced polarization density. It is obvious using (43) that $\mathbf{P}_{L}^{\prime}$ is transverse field.

\section{E. New Lagrangian}

With the Lagrangian in the form (33) we can substitute the expression (52) for the vector potential enabling the Lagrangian to be given in terms of the generalized field velocities $\dot{q}_{k}\left(k\right.$ in half space) and the particle velocities $\dot{\mathbf{r}}_{\xi \alpha}$. We find that

$$
\begin{aligned}
L^{\prime}= & \sum_{\xi \alpha} \frac{1}{2} M_{\xi \alpha} \dot{\mathbf{r}}_{\xi \alpha}^{2}-V_{\mathrm{Coul}}+\sum_{k}{ }^{\prime} \dot{q}_{k} \dot{q}_{k}^{*}-\sum_{k}{ }^{\prime} \omega_{k}^{2} q_{k} q_{k}^{*}+\sum_{\xi \alpha} \dot{\mathbf{r}}_{\xi \alpha} \cdot \int_{0}^{1} u d u B\left(\mathbf{R}_{\xi}+u\left(\mathbf{r}_{\alpha \xi}-\mathbf{R}_{\xi}\right)\right) \times\left(\mathbf{r}_{\alpha \xi}-\mathbf{R}_{\xi}\right) q_{\xi \alpha} \\
& -\sum_{k}{ }^{\prime}\left(\dot{q}_{k} \int d^{3} \mathbf{R} \mathbf{P}_{L} \cdot \mathbf{A}_{k}+\dot{q}_{k}^{*} \int d^{3} \mathbf{R} \mathbf{P}_{L} \cdot \mathbf{A}_{k}^{*}\right) .
\end{aligned}
$$

The details are given in Appendix D.

\section{F. Conjugate momenta}

The conjugate momenta are obtained from the Lagrangian (56) via the use of (38). The particle momenta $\mathbf{p}_{\xi \alpha}$ are, of course, as in (34). The generalized momentum coordinate $p_{k}$ for the field is given by

$$
p_{k}=\dot{q}_{k}-\int d^{3} \mathbf{R} \mathbf{P}_{L} \cdot \mathbf{A}_{k}^{*}
$$

and this can be extended to the full space via the use of (46) and (51) to give

$$
p_{k} *\left(p_{k}\right)^{*} \text {. }
$$

\section{G. Conjugate momentum field}

The expression for the conjugate momentum field can be determined from (35), (53), and (57) as follows:

$$
\begin{aligned}
\boldsymbol{\pi} & =\varepsilon \dot{\mathbf{A}}-\mathbf{P}_{L}^{\prime} \\
& =\sum_{k} \varepsilon \mathbf{A}_{k}\left(\dot{q}_{k}-\int d^{3} \mathbf{R} \mathbf{P}_{L} \cdot \mathbf{A}_{k}^{*}\right) \\
& =\sum_{k} p_{k} \varepsilon \mathbf{A}_{k} \\
& =\sum_{k}{ }^{\prime}\left(p_{k} \varepsilon \mathbf{A}_{k}+p_{k}^{*} \varepsilon \mathbf{A}_{k}^{*}\right)
\end{aligned}
$$

Thus we see that the field momentum is a linear combination of the $\varepsilon \mathbf{A}_{k}$ with the generalized momentum coordinates $p_{k}$ as coefficients. We note that the conjugate momentum field $\pi$ is always transverse

$$
\boldsymbol{\nabla} \cdot \boldsymbol{\pi}=0
$$

\section{H. New multipolar Hamiltonian}

This can be obtained in terms of the generalized coordinates $q_{k}$ and momenta $p_{k}$ for the field. Using the general result (39) and the Lagrangian given by (56), we then substitute for the particle velocities $\dot{\mathbf{r}}_{\xi \alpha}$ and generalized field velocities $\dot{q}_{k}$ via (34) and (57). The details are given in Appendix E. It is convenient to write the result in terms of $k$ sums over the full space and we find that

$$
\begin{aligned}
H^{\prime}= & \sum_{\xi \alpha} \frac{\mathbf{p}_{\xi \alpha}^{2}}{2 M_{\xi \alpha}}+V_{\text {Coul }}+\sum_{k} \frac{1}{2}\left|\int d^{3} \mathbf{R} \mathbf{P}_{L} \cdot \mathbf{A}_{k}\right|^{2} \\
& +\sum_{k} \frac{1}{2}\left(p_{k} p_{k}^{*}+\omega_{k}^{2} q_{k} q_{k}^{*}\right)+\sum_{k} p_{k} \int d^{3} \mathbf{R} \mathbf{P}_{L} \cdot \mathbf{A}_{k} \\
& -\sum_{k} q_{k} \int d^{3} \mathbf{R} \mathbf{M}_{L}^{\prime} \cdot \boldsymbol{\nabla} \times \mathbf{A}_{k}+\sum_{\xi \alpha} \frac{q_{\xi \alpha}^{2}}{2 M_{\xi \alpha}} \\
& \times\left(\sum_{k} q_{k} \int_{0}^{1} u d u \boldsymbol{\nabla} \times \mathbf{A}_{k}\left[\mathbf{R}_{\xi}+u\left(\mathbf{r}_{\xi \alpha}-\mathbf{R}_{\xi}\right)\right]\right. \\
& \left.\times\left(\mathbf{r}_{\xi \alpha}-\mathbf{R}_{\xi}\right)\right)^{2} .
\end{aligned}
$$


In order the terms in the Hamiltonian (61) are particle kinetic energy, Coulomb energy, polarization energy, radiation field energy, electric interaction energy, magnetic interaction energy, and diamagnetic energy. The radiation field energy is the sum of independent harmonic oscillators terms for each mode, with $\omega_{k}$ acting as the oscillator frequency. The electric and magnetic interaction energies depend linearly on the generalized momenta $p_{k}$ and generalized coordinates $q_{k}$, respectively. The diamagnetic energy depends quadratically on the generalized coordinates. It should be noted that this generalized multipolar Hamiltonian is restricted to the case of neutral, stationary, radiative atoms with an electrically neutral nonconducting dielectric and magnetic medium. The Hamiltonian is still classical at this stage.

Another form of the multipolar Hamiltonian can be obtained from the original result (36) by expanding the term involving $\left(\boldsymbol{\pi}+\mathbf{P}_{L}^{\prime}\right)^{2}$. This is given as

$$
\begin{aligned}
H^{\prime}= & \sum_{\xi \alpha} \frac{\mathbf{p}_{\xi \alpha}^{2}}{2 M_{\xi \alpha}}+V_{\mathrm{Coul}}+\int d^{3} \mathbf{R} \frac{\left[\mathbf{P}_{L}^{\prime}(\mathbf{R})\right]^{2}}{2 \varepsilon} \\
& +\int d^{3} \mathbf{R}\left(\frac{\boldsymbol{\pi}^{2}}{2 \varepsilon}+\frac{(\boldsymbol{\nabla} \times \mathbf{A})^{2}}{2 \mu}\right) \\
& +\int d^{3} \mathbf{R} \frac{\boldsymbol{\pi} \cdot \mathbf{P}_{L}^{\prime}}{\varepsilon}-\int d^{3} \mathbf{R}(\boldsymbol{\nabla} \times \mathbf{A}) \cdot \mathbf{M}_{L}^{\prime}+\sum_{\xi \alpha} \frac{q_{\xi \alpha}^{2}}{2 M_{\xi \alpha}} \\
& \times\left(\int_{0}^{1} u d u \mathbf{B}\left(\mathbf{R}_{\xi}+u\left(\mathbf{r}_{\xi \alpha}-\mathbf{R}_{\xi}\right)\right) \times\left(\mathbf{r}_{\xi \alpha}-R_{\xi}\right)\right)^{2} .
\end{aligned}
$$

Using the mode expansions (49), (59), and (53) for $\mathbf{A}(\mathbf{R})$, $\boldsymbol{\pi}(\mathbf{R})$, and $\mathbf{P}_{L}^{\prime}(\mathbf{R})$, respectively, it is easily shown, using the orthogonality condition (45), that the terms in (62) are equal to the corresponding terms in (61). Thus, as in (61), the terms in (62) in order are particle kinetic energy, Coulomb energy, polarization energy, radiation field energy, electric interaction energy, magnetic interaction energy, and diamagnetic energy. We note that the radiation field energy involves the square of the conjugate momentum field $\boldsymbol{\pi}$ as well as the square of the magnetic field $\mathbf{B}$. The electric interaction energy involves the conjugate momentum field $\boldsymbol{\pi}$ and the reduced polarization density $\mathbf{P}_{L}^{\prime}$. The magnetic interaction energy involves the magnetic field $\mathbf{B}$ and the reduced magnetization density $\mathbf{M}_{L}^{\prime}$. The diagmagnetic energy is quadratic in the magnetic field $\mathbf{B}$.

\section{GENERALIZED QUANTUM MULTIPOLAR HAMILTONIAN}

\section{A. Canonical quantization}

In accordance with the canonical quantization procedure [1] we replace the classical quantities with quantum mechanical operators. For the radiative atoms the positions and momenta for the charged particles are represented by Hermitian operators

$$
\begin{aligned}
\mathbf{r}_{\xi \alpha} \rightarrow \hat{\mathbf{r}}_{\xi \alpha}, \\
\mathbf{p}_{\xi \alpha} \rightarrow \hat{\mathbf{p}}_{\xi \alpha}
\end{aligned}
$$

with the usual commutation rules applying, for which the nonzero commutators are $(i, j=x, y, z)$

$$
\left[\hat{r}_{\xi \alpha i}, \hat{p}_{\eta \beta j}\right]=i \hbar \delta_{\xi \eta} \delta_{\alpha \beta} \delta_{i j}
$$

For the field we initially restrict ourselves to the half space and replace the generalized coordinates $q_{k}$ and the generalized momenta $p_{k}$ and their complex conjugates by nonHermitian operators

$$
\begin{aligned}
& q_{k} \rightarrow \hat{q}_{k}, \\
& q_{k}^{*} \rightarrow \hat{q}_{k}^{\dagger}, \\
& p_{k} \rightarrow \hat{p}_{k}, \\
& p_{k}^{*} \rightarrow \hat{p}_{k}^{\dagger} .
\end{aligned}
$$

Thus the complex conjugates are replaced by the Hermitian adjoints. The standard commutation rules apply, for which the nonzero commutators are

$$
\left[\hat{q}_{k}, \hat{p}_{l}^{\dagger}\right]=\left[\hat{q}_{k}^{\dagger}, \hat{p}_{l}\right]=i \hbar \delta_{k l}
$$

for $k, l$ in the half space. Note that the fundamental commutator which is nonzero involves $\hat{q}_{k}$ and $\hat{p}_{k}^{\dagger}$ and not $\hat{p}_{k}$. It is convenient to extend the relationships into the full space via the use of (51),(58) so that

$$
\begin{aligned}
& q_{k *} \rightarrow \hat{q}_{k}^{\dagger}, \\
& p_{k *} \rightarrow \hat{p}_{k}^{\dagger},
\end{aligned}
$$

where $k^{*}$ will be in the other half space. By this means the commutator rules (66) can be extended to apply to all $k, l$ and we have the relationship

$$
\begin{array}{r}
\hat{q}_{k *}=\hat{q}_{k}^{\dagger}, \\
\hat{p}_{k^{*}}=\hat{p}_{k}^{\dagger}
\end{array}
$$

between the operators representing the $k^{*}$ and $k$ modes.

\section{B. Annihilation and creation operators}

Since the radiation field energy is equivalent to that of a set of harmonic oscillators it is appropriate to introduce annihilation and creation operators for the quantum description of the field. These are defined via

$$
\begin{aligned}
& \hat{a}_{k}=\sqrt{\frac{\omega_{k}}{2 \hbar}} \hat{q}_{k}+i \frac{1}{\sqrt{2 \hbar \omega_{k}}} \hat{p}_{k}, \\
& \hat{a}_{k}^{\dagger}=\sqrt{\frac{\omega_{k}}{2 \hbar}} \hat{q}_{k}^{\dagger}-i \frac{1}{\sqrt{2 \hbar \omega_{k}}} \hat{p}_{k}^{\dagger},
\end{aligned}
$$


where $\hat{a}_{k}, \hat{a}_{k}^{\dagger}$ are the annihilation and creation operators, respectively. Again, these expressions can be taken to apply to all $k$. The usual commutation rules apply, where the nonzero commutators are given by

$$
\left[\hat{a}_{k}, \hat{a}_{l}^{\dagger}\right]=\delta_{k l}
$$

The definitions of the annihilation and creation operators can be inverted to give

$$
\begin{gathered}
\hat{q}_{k}=\sqrt{\frac{\hbar}{2 \omega_{k}}}\left(\hat{a}_{k}+a_{k^{*}}^{\dagger}\right), \\
\hat{p}_{k}=\frac{1}{i} \sqrt{\frac{\hbar \omega_{k}}{2}}\left(\hat{a}_{k}-\hat{a}_{k *}^{\dagger}\right),
\end{gathered}
$$

showing that the generalized coordinate $\hat{q}_{k}$ and generalized momenta $\hat{p}_{k}$ are related to the annihilation operator for the $k$ mode and the creation operator for the $k^{*}$ mode.

\section{Vector potential and conjugate momenta field}

The vector potential $\mathbf{A}(\mathbf{R})$ and the conjugate momentum field $\boldsymbol{\pi}(\mathbf{R})$ become quantum field operators via the replacement of the $q_{k}, p_{k}$ in the mode expansions $(49),(59)$ via the quantum operators $\hat{q}_{k}, \hat{p}_{k}$

$$
\begin{aligned}
\mathbf{A} \rightarrow \hat{\mathbf{A}}=\sum_{k} \hat{q}_{k} \mathbf{A}_{k}=\sum_{k} \sqrt{\frac{\hbar}{2 \omega_{k}}}\left[\hat{a}_{k} \mathbf{A}_{k}(R)+\hat{a}_{k}^{\dagger} \mathbf{A}_{k}^{*}(R)\right], \\
\boldsymbol{\pi} \rightarrow \hat{\boldsymbol{\pi}}=\sum_{k} \hat{p}_{k} \varepsilon A_{k}=\sum_{k}\left(\frac{1}{i} \sqrt{\frac{\hbar \omega_{k}}{2}} \hat{a}_{k} \varepsilon \mathbf{A}_{k}(R)\right. \\
\left.-\frac{1}{i} \sqrt{\frac{\hbar \omega_{k}}{2}} \hat{a}_{k}^{\dagger} \boldsymbol{\varepsilon} \mathbf{A}_{k}^{*}(R)\right) .
\end{aligned}
$$

The second forms of $\hat{\mathbf{A}}$ and $\hat{\boldsymbol{\pi}}$ follow from the expression (71) for the $\hat{q}_{k}, \hat{p}_{k}$ in terms of annihilation and creation operators. The field operators $\hat{\mathbf{A}}(\mathbf{R})$ and $\hat{\boldsymbol{\pi}}(\mathbf{R})$ are Hermitian. We can now obtain the commutation rules for $\hat{\mathbf{A}}, \hat{\boldsymbol{\pi}}$. Obviously the components of $\hat{\mathbf{A}}$ all commute, as do those of $\hat{\pi}$, since all $\hat{q}_{k}$ commute and all $\hat{p}_{k}$ commute. The commutators which are nonzero are

$$
\begin{aligned}
{\left[\hat{A}_{i}(\mathbf{R}), \hat{\pi}_{j}\left(\mathbf{R}^{\prime}\right)\right]=} & \sum_{k l} A_{k i}(\mathbf{R}) A_{l j}^{*}\left(\mathbf{R}^{\prime}\right) \varepsilon\left(R^{\prime}\right)\left[\hat{q}_{k}, \hat{p}_{l}^{\dagger}\right] \\
= & i \hbar \sum_{k} A_{k i}(\mathbf{R}) A_{k j}^{*}\left(\mathbf{R}^{\prime}\right) \varepsilon\left(\mathbf{R}^{\prime}\right) \\
& (i, j=x, y, z),
\end{aligned}
$$

which is a generalization of the result involving the transverse $\delta$ function $\left[i \hbar \delta_{i j}^{\perp}\left(\mathbf{R}-\mathbf{R}^{\prime}\right)\right]$ for the free field case [1]. It is hard to see how this result could have been just postulated, indicating the necessity of a canonical quantization procedure involving the mode functions and invoking the basic commutation rules (66).

\section{Other field operators} by

The magnetic field $\mathbf{B}$ also becomes a field operator given

$$
\hat{\mathbf{B}}(\mathbf{R})=\nabla \times \hat{\mathbf{A}}(\mathbf{R}) .
$$

The electric field $\mathbf{E}$ can be expressed as a field operator using (35) and (14) to give

$$
\hat{\mathbf{E}}(\mathbf{R})=-\left(\frac{\hat{\boldsymbol{\pi}}(\mathbf{R})+\hat{\mathbf{P}}_{L}^{\prime}(\mathbf{R})}{\varepsilon(\mathbf{R})}\right)-\nabla \hat{\phi}(\mathbf{R}),
$$

where both $\hat{P}_{L}^{\prime}$ and $\boldsymbol{\nabla} \hat{\phi}$ can be considered as operators depending on the radiative charged particle position operators $\hat{r}_{\xi \alpha}$.

There is no simple relationship between the transverse component of the electric displacement $\hat{\mathbf{D}}$ and $\hat{\boldsymbol{\pi}}$ as in the case for a free field. From the condition that

$$
\boldsymbol{\nabla} \cdot \hat{\mathbf{D}}=\hat{\rho}_{0}
$$

then the situation of neutral dielectric and radiative atoms $\left(\rho_{\mathrm{L} 0}=\rho_{\mathrm{D} 0}=0\right)$ implies that $\rho_{0}=0$, so that $\hat{\mathbf{D}}$ is purely transverse

$$
\hat{\mathbf{D}}=\hat{\mathbf{D}}_{\perp}
$$

It is then easy to see from (11), (12), and (76) that

$$
\hat{\mathbf{D}}=\hat{\mathbf{D}}_{\perp}=-\hat{\boldsymbol{\pi}}-(\varepsilon \nabla \hat{\boldsymbol{\phi}})_{\perp}+\left(\hat{\mathbf{P}}_{L}\right)_{\perp}-\mathbf{P}_{L}^{\prime}
$$

Thus in general $\hat{\mathbf{D}}_{\perp}$ and $-\hat{\boldsymbol{\pi}}$ are not equal. For the free space situation $\varepsilon \nabla \hat{\phi}=\varepsilon_{0} \boldsymbol{\nabla} \hat{\phi}$, which is purely longitudinal, $\mathbf{P}_{L}^{\prime}$ $=\left(\hat{\mathbf{P}}_{L}\right)_{\perp}$, so that $\hat{\mathbf{D}}=-\hat{\boldsymbol{\pi}}$.

\section{E. Quantum Hamiltonian}

The classical multipolar Hamiltonian (62) is replaced by the quantum multipolar Hamiltonian via substituting the classical quantities $\mathbf{P}_{L}^{\prime}, \mathbf{M}_{L}^{\prime}, \mathbf{A}, \boldsymbol{\pi}$ by the field operators and replacing the classical charged particle position and momentum coordinates $\mathbf{r}_{\xi \alpha}, \mathbf{P}_{\xi \alpha}$ by the quantum operators. For convenience the radiation field energy term is written in terms of annihilation and creation operators. Details are given in Appendix F. We find that the generalized quantum multipolar Hamiltonian for the case of neutral, stationary radiative atoms in a neutral, nonconducting, dielectric and magnetic medium is given by 


$$
\begin{aligned}
\hat{H}^{\prime}= & \sum_{\xi \alpha} \frac{\hat{\mathbf{p}}_{\xi \alpha}^{2}}{2 M_{\xi \alpha}}+\hat{V}_{\mathrm{Coul}}+\int d^{3} \mathbf{R} \frac{\left(\hat{\mathbf{P}}_{L}^{\prime}\right)^{2}}{2 \varepsilon}+\sum_{k}\left(\hat{a}_{k}^{\dagger} \hat{a}_{k}+\frac{1}{2}\right) \hbar \omega_{k}+\int d^{3} \mathbf{R} \frac{\hat{\mathbf{P}}_{L}^{\prime} \cdot \hat{\boldsymbol{\pi}}}{\varepsilon}-\int d^{3} \mathbf{R} \hat{\mathbf{M}}_{L}^{\prime} \cdot \boldsymbol{\nabla} \times \hat{\mathbf{A}} \\
& +\sum_{\xi \alpha} \frac{q_{\xi \alpha}^{2}}{2 M_{\xi \alpha}}\left(\int_{0}^{1} u d u \hat{B}\left(\mathbf{R}_{\xi}+u\left(\mathbf{r}_{\xi \alpha}-\mathbf{R}_{\xi}\right)\right) \times\left(\mathbf{r}_{\xi \alpha}-\mathbf{R}_{\xi}\right)\right)^{2} .
\end{aligned}
$$

In order the terms of the Hamiltonian are particle kinetic energy, Coulomb energy, polarization energy, radiation field energy, electric interaction energy, magnetic interaction energy, and diamagnetic energy. This quantum Hamiltonian is based on a vector mode function treatment. We see that the form of the radiation field energy establishes the photon picture for this system, with the quantum field being equivalent to a set of quantum harmonic oscillators, one for each mode of the field. It should be noted that there are no direct coupling terms between the different modes, a characteristic feature associated with the use of the exact vector mode functions associated with the classical linear optics device ("true modes"). The electric and magnetic interaction energy terms allow for basic atom-field processes in which one photon is created or destroyed in each fundamental step, this being a consequence of the linear dependence of these interaction energy terms on the creation and annihilation operators.

\section{F. Electric dipole approximation}

To obtain the electric dipole approximation result we ignore the polarization energy, magnetic interaction energy, and diamagnetic energy terms in the quantum Hamiltonian (80) and replace the polarization density with its dipolar approximation

$$
\hat{\mathbf{P}}_{L}(\mathbf{R})=\sum_{\xi} \hat{\boldsymbol{\mu}}_{\xi} \delta\left(\mathbf{R}-\mathbf{R}_{\xi}\right)
$$

where $\hat{\boldsymbol{\mu}}_{\xi}$ is the dipole operator for the $\xi$ atom. In view of (31) the $\mathbf{R}_{\xi}$ are classical. The transverse $\left(\hat{\mathbf{P}}_{L}\right)_{\perp}$ and longitudinal $\left(\hat{\mathbf{P}}_{L}\right)_{\|}$components of $\hat{P}_{L}$ may be evaluated using the Helmholtz theorem [10]. These are given as (details are in Appendix G)

$$
\begin{aligned}
\left(\hat{\mathbf{P}}_{L}(\mathbf{R})\right)_{\perp} & =\frac{-1}{4 \pi} \sum_{\xi}\left(\frac{\hat{\boldsymbol{\mu}}_{\xi}}{\left|\mathbf{R}-\mathbf{R}_{\xi}\right|^{3}}-\frac{3\left(\mathbf{R}-\mathbf{R}_{\xi}\right) \cdot \hat{\boldsymbol{\mu}}_{\xi}\left(\mathbf{R}-\mathbf{R}_{\xi}\right)}{\left|\mathbf{R}-\mathbf{R}_{\xi}\right|^{5}}\right) \\
& =-\left(\hat{\mathbf{P}}_{L}(\mathbf{R})\right)_{\|} \text {for }\left(\mathbf{R} \neq \mathbf{R}_{\xi}\right) .
\end{aligned}
$$

A straightforward calculation gives the reduced polarization density as

$$
\hat{\mathbf{P}}_{L}^{\prime}(\mathbf{R})=\sum_{k \xi} \hat{\boldsymbol{\mu}}_{\xi} \cdot \mathbf{A}_{k}^{*}\left(\mathbf{R}_{\xi}\right) \varepsilon(\mathbf{R}) \mathbf{A}_{k}(\mathbf{R})
$$

and substituting for $\hat{\boldsymbol{\pi}}(\mathbf{R})$ in terms of the mode expression (73) enables the Hamiltonian to be determined. The different results for the transverse component of $\hat{\mathbf{P}}_{L}(\mathbf{R})$ and the reduced polarization density $\hat{\mathbf{P}}_{L}^{\prime}(\mathbf{R})$ are apparent.

The quantum Hamiltonian in the electric dipole approximation is given by

$$
\begin{aligned}
\hat{H}_{E 1}^{\prime}= & \sum_{\xi \alpha} \frac{\mathbf{p}_{\xi \alpha}^{2}}{2 M_{\xi \alpha}}+\hat{V}_{\mathrm{Coul}}+\sum_{k}\left(\hat{a}_{k}^{\dagger} \hat{a}_{k}+\frac{1}{2}\right) \hbar \omega_{k} \\
& +\sum_{\xi \alpha} \sqrt{\frac{\hbar \omega_{k}}{2}} \frac{1}{i}\left[\hat{a}_{k} \hat{\boldsymbol{\mu}}_{\xi} \cdot \mathbf{A}_{k}\left(\mathbf{R}_{\xi}\right)-\hat{a}_{k}^{\dagger} \hat{\boldsymbol{\mu}}_{\xi} \cdot \mathbf{A}_{k}^{*}\left(\mathbf{R}_{\xi}\right)\right],
\end{aligned}
$$

where the first two terms give the particle energy, the third term is the radiation field energy, and the last term is the electric dipole interaction energy.

Thus the atom-field interaction is given by

$\hat{H}_{\mathrm{AF}}=\sum_{k \xi} \sqrt{\frac{\hbar \omega_{k}}{2}} \frac{1}{i}\left[\hat{a}_{k} \hat{\boldsymbol{\mu}}_{\xi} \cdot \mathbf{A}_{k}\left(\mathbf{R}_{\xi}\right)-\hat{a}_{k}^{\dagger} \hat{\boldsymbol{\mu}}_{\xi} \cdot \mathbf{A}_{k}^{*}\left(\mathbf{R}_{\xi}\right)\right]$

This form has been widely used in cavity QED calculations [11]. It involves the sum over modes and radiative atoms and includes scalar products of the mode functions evaluated at the atomic center of mass $\mathbf{R}_{\xi}$ with the atomic dipole operators, as well as the mode creation and annihilation operators and the factor $\left(\hbar \omega_{k} / 2\right)^{1 / 2}$ giving the electric field per photon. The atom-field interaction can also be written in the form

$$
\hat{H}_{\mathrm{AF}}=\sum_{\xi} \frac{\hat{\boldsymbol{\mu}}_{\xi} \cdot \hat{\boldsymbol{\pi}}\left(\mathbf{R}_{\xi}\right)}{\varepsilon\left(\mathbf{R}_{\xi}\right)}
$$

displaying the dependence on the conjugate momentum field evaluated at the atomic center of mass. Note that because $\hat{\boldsymbol{\tau}} / \varepsilon$ is not the same as $-\hat{\mathbf{D}}_{\perp} / \varepsilon$, this form cannot be simply related to the transverse component of the electric displacement operator, as in the free field case.

\section{SIMPLE APPLICATION ATOMIC DECAY IN A ONE-DIMENSIONAL CAVITY}

As a simple application we consider the decay of an excited and level atom placed at the center of a onedimensional cavity of length $l$ (see Fig. 2). The cavity is of the Fabry-Perot type: one mirror is perfect, the other is made up of a dielectric layer of thickness $d$ with permittivity $\varepsilon_{1}=\kappa_{1} \varepsilon_{0}, \kappa_{1}$ being the dielectric constant. The region external to the cavity is of length $L$ and a second perfect mirror is situated at its end. The cross sectional area of the system is $\mathcal{A}$. Such cavities, in which the field and radiating atoms are contained in a one-dimensional universe between the two perfect mirrors have often been used for laser theory; see, for example, Lang et al. [12]. In the present case the decay of an excited atom will be treated, via an approach of Lai et al. 
[13], and which demonstrates the full range of behavior from cavity modified irreversible spontaneous emission in the weak coupling regime to reversible energy interchange between atom and field in the strong coupling regime.

In the electric dipole approximation (85) the atom-field coupling can be written

$$
\hat{H}_{\mathrm{AF}}=\sum_{k} \hbar g_{k} \hat{S} \hat{a}_{k}+(\text { Hermitian conjugate }),
$$

where the coupling constant $g_{k}$ is given by

$$
g_{k}=\frac{1}{i} \sqrt{\frac{\omega_{k}}{2 \hbar}} \boldsymbol{\mu} \cdot \mathbf{A}_{k}(\mathbf{R}) .
$$

$\boldsymbol{\mu}$ is the dipole matrix element $\langle 2|\hat{\boldsymbol{\mu}}| 1\rangle, \hat{S}=|2\rangle\langle 1|$ is the usual atomic transition operator. $|2\rangle,|1\rangle$ are the atomic excited- and ground-state eigenvectors. The atom is at position $\mathbf{R}$, where the mode function is $\mathbf{A}_{k}(\mathbf{R})$ for mode $k$.

The form of the mode functions inside this cavity is derived in Appendix $\mathrm{H}$ and is given by

$$
\mathbf{A}_{k}(\mathbf{R})=\mathbf{i} \sqrt{\frac{2}{\varepsilon_{0} L \mathcal{A}}} \frac{\Lambda_{n} \Gamma_{n}}{\sqrt{\left(k-k_{n}\right)^{2}+\Gamma_{n}^{2}}} \sin k(z-l), \quad 0 \leqslant z \leqslant l
$$

assuming $\left|k-k_{n}\right| \ll \Gamma_{n} \Lambda_{n}$. Here $\Lambda_{n}, \Gamma_{n}$ are peak height and width factors for the cavity, expressions for which are

$$
\begin{gathered}
\Lambda_{n}=\kappa_{1} k_{n} d, \\
\Gamma_{n}=\frac{1}{\Lambda_{n}^{2} l} .
\end{gathered}
$$

The wave vector $k_{n}$ for the $n$th Fabry-Pérot resonance is given by

$$
k_{n} \approx n \frac{\pi}{l}+\frac{1}{l \Lambda_{n}}, \quad n=0,1,2, \ldots
$$

In these expressions the thin strong dielectric regime applies (see Appendix H) where $\sqrt{\kappa_{1}} k d \ll 1, \kappa_{1} k d \gg 1$, and where the mode separation $\Delta k \sim \pi / L$ is small compared to the width factor $\Gamma_{n}$. The mode function has essentially a Lorentzian dependence on wave number $k$, with $\Lambda_{n}(\gg 1)$ defining the peak height and $\Gamma_{n}$ the width.

Taking the dipole operator along $\mathbf{i}$ and with the atom at $z=l / 2$, the coupling constant $g_{k}$ is given by

$$
g_{k}=i \sqrt{\frac{\omega_{k}}{\hbar \varepsilon_{0} L \mathcal{A}}} \mu \frac{\Gamma_{n} \Lambda_{n}}{\sqrt{\left(k-k_{n}\right)^{2}+\Gamma_{n}^{2}}} \sin \frac{1}{2} k l .
$$

The atom is initially in excited state $|2\rangle$ and there are no photons in the field. An essential states approach for a timedependent state vector $\left|\Phi_{I}\right\rangle$ in the interaction picture is used to describe the process in which the atom makes a transition to the ground state $|1\rangle$ and one photon appears in one of the field modes $k$. The state vector $\left|\Phi_{I}\right\rangle$ is written as

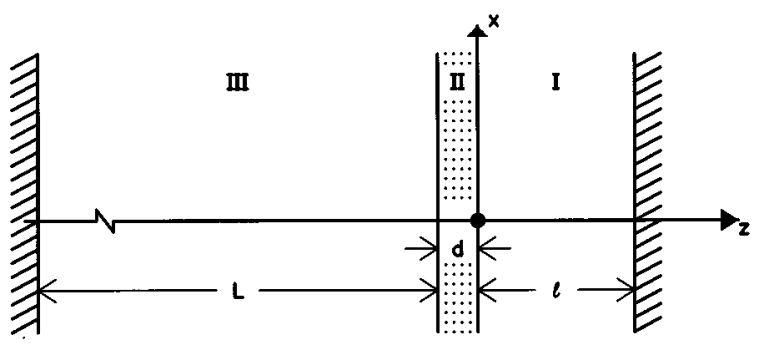

FIG. 2. One-dimensional cavity model of the Fabry-Perot type. Regions I, II, and III are the cavity, dielectric, and external regions, respectively. The hatched regions at the ends are perfectly reflecting mirrors. The cross-sectional area is $\mathcal{A}$.

$$
\left.\left|\varphi_{1}\right\rangle=c_{2}|2\rangle\left|\cdots 0_{k} \cdots\right\rangle+\sum_{k} c_{1 k}|1\rangle \cdots 1_{k} \cdots\right\rangle, \quad t \geqslant 0,
$$

where $c_{2}, c_{1 k}$ are amplitudes for the states $|2\rangle\left|\cdots 0_{k} \cdots\right\rangle$ and $\left.|1\rangle \mid \cdots 1_{k} \cdots\right)$, respectively.

The coupled amplitude equations are

$$
\begin{gathered}
\dot{c}_{2}=-i \sum_{k} g_{k} e^{i \delta_{k} t} c_{1 k}, \\
\dot{c}_{1 k}=i g_{k}^{*} e^{-i \delta_{k} t} c_{2},
\end{gathered}
$$

with

$$
\delta_{k}=\omega_{0}-\omega_{k},
$$

and where $\omega_{0}$ is the atomic transition frequency.

Formally solving the second amplitude equation with initial conditions $c_{2}(0)=1, c_{1 k}(0)=0$ and substituting the solution for $c_{1 k}$ into the first amplitude equation leads to an integrodifferential equation for the probability amplitude $c_{2}$ that the atom remains excited, given as

$$
\frac{d c_{2}(t)}{d t}=\int_{0}^{t} d \tau K(\tau) c_{2}(t-\tau) .
$$

The kernel $K(\tau)$ is given by

$$
K(\tau)=-\sum_{k}\left|g_{k}\right|^{2} e^{i \delta_{k} \tau}
$$

The solution may be obtained using Laplace transform methods in which a function $X(t) \quad(t \geqslant 0)$ and its Laplace transform $\widetilde{X}(s)$ are related via

$$
\begin{aligned}
& \widetilde{X}(s)=\int_{0}^{\infty} d t e^{-s t} X(t), \quad \operatorname{Re} s>0, \\
& X(t)=\frac{1}{2 \pi i} \int_{c} d s e^{+s t} \widetilde{X}(s), \quad t>0,
\end{aligned}
$$

where $c$ is a contour to the right of the imaginary axis going from $-i \infty$ to $+i \infty$. In the present case the Laplace transform for $c_{2}(t)$ is given in terms of the Laplace transform of $K(t)$ as 


$$
\widetilde{c}_{2}(s)=\frac{1}{s-\widetilde{K}(s)} .
$$

The properties of the kernel determines whether the atomic decay exhibits reversible (non-Markovian) or irreversible (Markovian) behavior. We find that

$$
\widetilde{K}(s)=i \sum_{k} \frac{\left|g_{k}\right|^{2}}{\left(\omega_{k}-\omega_{0}\right)-i s} .
$$

For a one-dimensional cavity model with $\omega_{n}=c k_{n}$ giving the cavity (Fabry-Perot) resonance frequencies the sum over $k$ can be evaluated based using the expression (92) for the coupling constants $g_{k}$ to give

$$
\begin{aligned}
\widetilde{K}(s) & =i \frac{\mu^{2} \omega_{n}}{\hbar \varepsilon_{0} \mathcal{A}} \frac{\Gamma_{n} \Lambda_{n}^{2} \nu_{n}}{s+c \Gamma_{n}+i\left(\omega_{n}-\omega_{0}\right)} \\
& =-\frac{1}{2} \Gamma_{0} \Lambda_{n}^{2} \frac{c \Gamma_{n} \nu_{n}}{s+c \Gamma_{n}+i\left(\omega_{n}-\omega_{0}\right)}
\end{aligned}
$$

where as before $\Gamma_{n}$ is the cavity width factor and $\Lambda_{n}$ is the cavity height factor, $\nu_{n}=0,1$ depending on whether the atom is at a node or antinode of the mode function, and where

$$
\Gamma_{0}=\frac{2 \mu^{2} \omega_{n}}{\hbar \varepsilon_{0} c \mathcal{A}}, \quad \omega_{n} \approx \omega_{0} .
$$

$\Gamma_{0}$ will be identified with the free space atomic spontaneous emission rate for this one-dimensional case. Inverting the Laplace transform gives the following expressions for the kernel $K(\tau)$ :

$$
K(\tau)=-\frac{1}{2} \Gamma_{0} \Lambda_{n}^{2} \nu_{n} c \Gamma_{n} e^{-i\left(\omega_{n}-\omega_{0}\right) \tau} e^{-c \Gamma_{n} \tau}
$$

This is an oscillating, decaying exponential with a correlation time equal to cavity decay time

$$
\tau_{c}=\left(c \Gamma_{n}\right)^{-1} .
$$

Markovian or non-Markovian behavior depends on whether this correlation time is short or long compared to the characteristic time over which $c_{2}(t)$ changes (still to be determined).

For the Markovian regime, we assume that $c_{2}$ hardly changes during correlation time $\tau_{c}$, the assumption to be checked a posterioi. Then

$$
\begin{aligned}
\dot{c}_{2} & \approx\left(\int_{0}^{\infty} d \tau K(\tau)\right) c_{2}(t) \quad \text { for } t \gg \tau_{c} \\
& \approx \widetilde{K}(\varepsilon) c_{2},
\end{aligned}
$$

where $\varepsilon$ is a small quantity.

Now

$$
\widetilde{K}(\varepsilon)=-\frac{1}{2} \Gamma_{0} \Lambda_{n}^{2} \nu_{n} \frac{c \Gamma_{n}}{c \Gamma_{n}+i\left(\omega_{n}-\omega_{0}\right)},
$$

giving the excited-state amplitude as

$$
c_{2}(t)=\exp \left\{-\frac{1}{2} \Gamma_{0} \Lambda_{n}^{2} \nu_{n} \frac{\Gamma_{n}}{i\left(k_{n}-k_{0}\right)+\Gamma_{n}} t\right\} .
$$

The excitation probability is therefore

$$
P_{2}(t)=\exp \left\{-\Gamma_{0} \Lambda_{n}^{2} \nu_{n} \frac{\Gamma_{n}^{2}}{\left(k_{n}-k_{0}\right)^{2}+\Gamma_{n}^{2}} t\right\} .
$$

The atom thus decays exponentially with time, but with a cavity modified atomic decay rate given by the Purcell [14] result

$$
\Gamma=\Gamma_{0} \Lambda_{n}^{2} \nu_{n} \frac{\Gamma_{n}^{2}}{\left(k_{n}-k_{0}\right)^{2}+\Gamma_{n}^{2}}
$$

Since $\Lambda_{n} \gg 1$ we see that the atomic decay rate is cavity enhanced from the free-space rate $\Gamma_{0}$ when the atom is at an antinode, and is cavity decreased (to zero) when the atom is at a node.

For the usual case of near resonance $\left(k_{n}-k_{0}\right) \sim \Gamma_{n}$ we see that the condition required for atomic Markovian behavior (irreversible decay) for an atom at an antinode is

$$
\Gamma_{0} \Lambda_{n}^{2} \tau_{c} \ll 1
$$

so that

$$
\frac{\Gamma_{0}}{c} \ll \frac{\Gamma_{n}}{\Lambda_{n}^{2}}
$$

Thus the free-space spontaneous emission width must be small compared to the cavity line width divided by the square of the peak height enhancement factor. This condition is the same as that required for the so-called weak-coupling regime, as will be seen later.

Returning to the general results given by (99) and (101) we find that the general solution for the Laplace transform $\widetilde{c_{2}}(s)$ is

$$
\widetilde{c}_{2}(s)=\frac{s+c \Gamma_{n}+i\left(\omega_{n}-\omega_{0}\right)}{s\left[s+c \Gamma_{n}+i\left(\omega_{n}-\omega_{0}\right)\right]+\frac{1}{2} \Gamma_{0} \Lambda_{n}^{2} \nu_{n} c \Gamma_{n}} .
$$

The behavior of the solution will depend on the poles of $\widetilde{c_{2}}(s)$ which are at the zeros of the denominator in (112) and are

$$
\begin{aligned}
s_{ \pm}= & -\frac{1}{2}\left[c \Gamma_{n}+i\left(\omega_{n}-\omega_{0}\right)\right] \\
& \pm \frac{1}{2} \sqrt{\left[c \Gamma_{n}+i\left(\omega_{n}-\omega_{0}\right)\right]^{2}+2 \Gamma_{0} \Lambda_{n}^{2} \nu_{n} c \Gamma_{n}}
\end{aligned}
$$

For simplicity we will only consider the antinode case for resonance where $k_{n}=k_{0}$, so that the atomic transition frequency coincides with a Fabry-Perot resonance. In this case

$$
s_{ \pm}=-\frac{1}{2} c \Gamma_{n} \pm \frac{1}{2} c \Gamma_{n} \sqrt{1-\frac{2 \Gamma_{0} \Lambda_{n}^{2}}{c \Gamma_{n}}}
$$

and 


$$
\widetilde{c_{2}}(s)=\frac{s+c \Gamma_{n}}{\left(s-s_{+}\right)\left(s-s_{-}\right)} .
$$

Two regimes may be distinguished depending on whether the poles $s_{ \pm}$are real (weak-coupling regime) or are complex (strong-coupling regime).

In the weak-coupling regime (poles real)

$$
\frac{\Gamma_{0}}{c} \ll \frac{\Gamma_{n}}{\Lambda_{n}^{2}},
$$

giving

$$
\begin{gathered}
s_{+} \approx-\frac{1}{2} c \Gamma_{n} \frac{\Gamma_{0} \Lambda_{n}^{2}}{c \Gamma_{n}} \approx-\frac{1}{2} \Gamma_{0} \Lambda_{n}^{2}, \\
s_{-} \approx-c \Gamma_{n}+\frac{1}{2} \Gamma_{0} \Lambda_{n}^{2} .
\end{gathered}
$$

In this case we find approximately that

$$
\begin{gathered}
c_{2}(t) \approx \exp \left(-\frac{1}{2} \Gamma_{n} \Lambda_{n}^{2} t\right), \\
P_{2}(t) \approx \exp \left(-\Gamma_{0} \Lambda_{n}^{2} t\right),
\end{gathered}
$$

which is the same solution as for the Markoff case, representing the overdamped regime with irreversible decay at the cavity modified decay rate $\Gamma_{0} \Lambda_{n}^{2}$.

In the strong-coupling regime (poles complex)

$$
\frac{\Gamma_{0}}{c} \gg \frac{\Gamma_{n}}{\Lambda_{n}^{2}},
$$

giving

$$
s_{ \pm} \approx-\frac{1}{2} c \Gamma_{n} \pm \frac{1}{2} i \sqrt{2 \Gamma_{0} \Lambda_{n}^{2} c \Gamma_{n}}
$$

In this case the expression for the probability amplitude $c_{2}$ is of the form

$$
\begin{aligned}
c_{2}(t)= & \left(\alpha e^{-(1 / 2) i \sqrt{2 \Gamma_{0} \Lambda_{n}^{2} c \Gamma_{n}} t}\right. \\
& +\alpha^{*} e^{\left.(1 / 2) i \sqrt{2 \Gamma_{0} \Lambda_{n}^{2} c \Gamma_{n} t}\right)} e^{-(1 / 2) c \Gamma_{n} t},
\end{aligned}
$$

where $\alpha$ is a constant. Hence the excitation probability will oscillate with a frequency

$$
\omega_{\mathrm{osc}}=\sqrt{2 \Gamma_{0} \Lambda_{n}^{2} c \Gamma_{n}}
$$

The excitation probability will also decay at the cavity decay rate $c \Gamma_{n}$.

Hence in this strong-coupling regime we have atomic non-Markovian behavior in an underdamped regime showing reversible photon emission into the cavity modes at an oscillation frequency $\omega_{\mathrm{osc}}$. In addition, there is a slow overall decay, corresponding to loss of energy from the cavity to the outside region.
In order to give an interpretation of $\omega_{\text {osc }}$ an effective onephoton Rabi frequency $g_{\text {eff }}$ can be introduced via summing the $\left(g_{k}\right)^{2}$ for all modes under the Fabry-Perot resonance

$$
g_{\text {eff }}^{2}=\sum_{k}\left|g_{k}\right|^{2}
$$

This gives

$g_{\text {eff }}=\frac{\omega_{0}}{\hbar \varepsilon_{0} \mathcal{A}} \mu \Lambda_{n} \Gamma_{n}^{1 / 2}=\frac{1}{2} \sqrt{2 \Gamma_{0} \Lambda_{n}^{2} c \Gamma_{n}}=\frac{1}{2} \omega_{\mathrm{osc}}$.

Thus the excitation probability oscillates at a rate given in terms of the effective one-photon Rabi frequency associated with replacing all the modes under the Fabry-Perot resonance by an equivalent single mode. This is the basis of the quasimode approach.

The criteria for the two regimes can also be stated in terms of the effective one-photon Rabi frequency;

$$
g_{\text {eff }} \ll c \Gamma_{n} \quad \text { (weak coupling) }
$$

and

$$
g_{\text {eff }} \gg c \Gamma_{n} \quad \text { (strong coupling). }
$$

Thus the weak-coupling regime has the cavity decay time much shorter than the atom-effective cavity mode interaction time, in the strong-coupling regime the atom and the effective cavity mode interchange energy much faster than it leaks out of the cavity. The behavior in the two regimes is shown in Fig. 3. These effects are, of course, quite well known, but serve as an illustrative application of the general results in this paper.

\section{CONCLUSIONS}

The key results may be summarized as follows. Macroscopic canonical quantization for the system of radiative atoms interacting with the EM field in the presence of a neutral dielectric medium describing passive, lossless, linear, classical optics devices has been carried out based on describing the dielectric atoms via an inhomogeneous electric permittivity and an inhomogeneous magnetic permeability. This canonical quantization procedure has been based on the exact vector mode functions associated with the classical optics device. The macroscopic canonical quantization leads to a generalized multipolar quantum Hamiltonian, containing terms corresponding to the particle kinetic energy, the Coulomb energy, the polarization energy, the radiation field energy, the electric interaction energy, the magnetic interaction energy, and the diamagnetic energy. The quantum Hamiltonian shows that the field is equivalent to a set of independent quantum harmonic oscillators, one for each mode, justifying the basic photon picture for this situation. The field oscillators are independent since there are no direct coupling terms between different modes in the Hamiltonian, and this arises because the exact vector mode functions for the optics device have been used. The atom-field coupling terms in the Hamiltonian show that the basic energy interchange processes involve one-photon emissions and absorptions. In the electric dipole approximation, the Hamiltonian is obtained in 


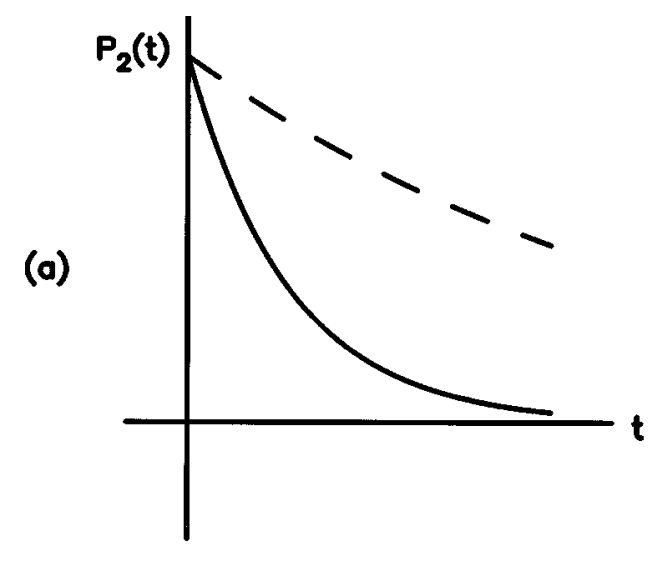

(b)

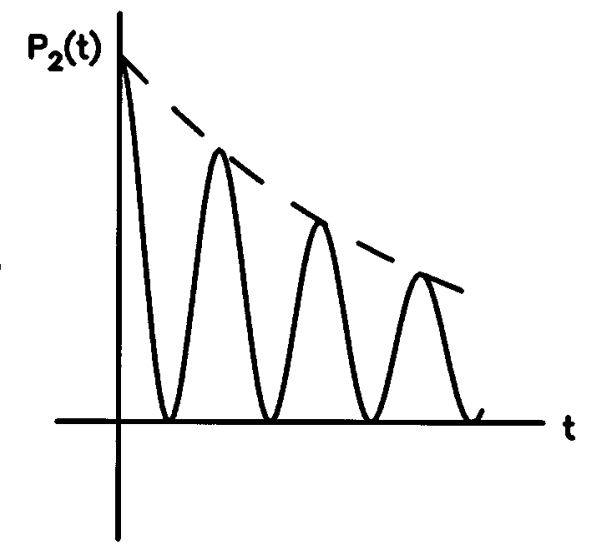

FIG. 3. Atomic excitation probability $P_{2}$ versus time $t$ for (a) the weak-coupling regime and (b) the strong-coupling regime. In both cases resonance conditions $k_{n}=k_{0}$ apply and the atom is at an antinode. In (b) the solid line indicates decay at the cavity modified rate $\Gamma_{n} \Lambda_{n}^{2}$, the dashed line indicates decay at the free space rate $\Gamma_{0}$. In (b) the solid line indicates the cavity modified oscillating decay, the dashed line indicates the cavity decay rate $c \Gamma_{n}$.

a form suitable for further applications. Here, the atom-field coupling terms for each field mode involve the scalar product of the dipole operator with the vector mode-function evaluated at the atomic center of mass, as well as annihilation and creation operators for the field mode. An illustrative application to atomic decay in a one-dimensional cavity has been given and the results are in accord with previous work.

\section{ACKNOWLEDGMENTS}

This work was supported by the Engineering and Physical Sciences Research Council (U.K.), the European Community, and CNPq. Helpful discussions with Dr. S. M. Barnett, Professor P. D. Drummond, Professor E. A. Hinds, Professor J. Kimble, Professor E. A. Power, Professor J. Sipe, and Professor S. Stenholm are gratefully acknowledged.

\section{APPENDIX A: UNIQUENESS OF A, $\varepsilon \nabla \phi$}

In the generalized radiation gauge we have

$$
\boldsymbol{\nabla} \cdot \varepsilon \mathbf{A}=0
$$

from which follows the generalized Poisson and the generalized inhomogeneous wave equations.

$$
\begin{gathered}
\boldsymbol{\nabla} \cdot(\varepsilon \boldsymbol{\nabla} \phi)=-\rho_{L}, \\
\boldsymbol{\nabla} \times \frac{1}{\mu}(\boldsymbol{\nabla} \times \mathbf{A})+\varepsilon \frac{\partial^{2}}{\partial t^{2}} \mathbf{A}=\mathbf{j}_{L}-\frac{\partial}{\partial t}(\varepsilon \boldsymbol{\nabla} \phi) .
\end{gathered}
$$

The various fields are given by

$$
\begin{gathered}
\mathbf{E}=-\boldsymbol{\nabla} \phi-\frac{\partial \mathbf{A}}{\partial t}, \\
\mathbf{B}=\boldsymbol{\nabla} \times \mathbf{A}, \\
\mathbf{D}=\varepsilon \mathbf{E}+\mathbf{P}_{L}, \\
\mathbf{H}=\frac{\mathbf{B}}{\mu}-\mathbf{M}_{L} .
\end{gathered}
$$

The Lagrangian in the minimal coupling form is

$$
L=\sum_{\xi \alpha} \frac{1}{2} M_{\xi \alpha} \dot{r}_{\xi \alpha}^{2}+\int d^{3} \mathbf{R}\left(\frac{1}{2} \varepsilon \mathbf{E}^{2}-\frac{1}{2 \mu} \mathbf{B}^{2}+\mathbf{j}_{L} \cdot \mathbf{A}-\rho_{L} \phi\right) .
$$

It is not obvious that $\mathbf{A}, \boldsymbol{\phi}$ are uniquely defined by the generalized radiation gauge condition and the generalized Poisson equation and generalized inhomogeneous wave equation. For example, the familiar conventional gauge transformations

$$
\begin{gathered}
\mathbf{A}^{\prime}=\mathbf{A}+\nabla \chi, \\
\phi^{\prime}=\phi-\dot{\chi}
\end{gathered}
$$

leave E,B unaltered. They alter the Lagrangian by $\boldsymbol{\nabla} \cdot\left(\mathbf{j}_{L} \chi\right)+\left(\partial / \partial_{t}\right)\left(\rho_{L} \chi\right)$, which leaves the action (and hence leaves the Maxwell and Lorentz equations) unchanged.

However other changes to $\mathbf{A}, \phi$ can be considered. The generalized radiation gauge condition would still be valid if a transverse field $\boldsymbol{\nabla} \times F$ was added to $\varepsilon \mathbf{A}$. Thus we may consider a possible vector potential, referred to as $\widetilde{\mathbf{A}}$ and given by

$$
\widetilde{\mathbf{A}}=\mathbf{A}+\frac{1}{\varepsilon} \boldsymbol{\nabla} \times \mathbf{F}
$$

Assuming that the magnetic field $\mathbf{B}$ is given by (A5) in terms of $\widetilde{\mathbf{A}}$, we require that

$$
\mathbf{B}=\boldsymbol{\nabla} \times \tilde{\mathbf{A}}=\boldsymbol{\nabla} \times \mathbf{A},
$$

so that

$$
\boldsymbol{\nabla} \times \frac{1}{\varepsilon}(\boldsymbol{\nabla} \times \mathbf{F})=0
$$

Also the electric field must be given by (A4) in terms of $\widetilde{\mathbf{A}}$ and a possible scalar potential referred to as $\widetilde{\phi}$ so that

$$
\mathbf{E}=-\dot{\widetilde{\mathbf{A}}}-\nabla \widetilde{\phi}=-\dot{\mathbf{A}}-\nabla \phi .
$$




$$
\varepsilon \nabla \widetilde{\phi}=\varepsilon \nabla \phi-\nabla \times \dot{\mathbf{F}} .
$$

This means that for the transverse and longitudinal components

$$
\begin{gathered}
(\varepsilon \nabla \widetilde{\phi})_{\|}=(\varepsilon \nabla \phi)_{\|}, \\
(\varepsilon \nabla \widetilde{\phi})_{\perp}=(\varepsilon \nabla \phi)_{\perp}-\nabla \times \dot{\mathbf{F}} .
\end{gathered}
$$

Obviously if $\mathbf{E}, \mathbf{B}$ are unaltered so also are $\mathbf{D}, \mathbf{H}$.

The generalized Poisson and inhomogeneous wave equations are still valid in terms of the new potentials. From (A16) and (A2)

$$
\boldsymbol{\nabla} \cdot(\varepsilon \nabla \widetilde{\phi})=\boldsymbol{\nabla} \cdot(\varepsilon \nabla \widetilde{\phi})_{\|}=\nabla \cdot(\varepsilon \nabla \phi)_{\|}=-\rho_{L} .
$$

Indeed the generalized Poisson equation itself only determines $(\varepsilon \nabla \phi)_{\|}$. In the free space situation, however, $\left(\varepsilon_{0} \boldsymbol{\nabla} \phi\right)_{\|}=\varepsilon_{0} \boldsymbol{\nabla} \phi$ since $\left(\varepsilon_{0} \boldsymbol{\nabla} \phi\right)_{\perp}=\varepsilon_{0}(\boldsymbol{\nabla} \phi)_{\perp}=0$. Also from (A3), (A11), (A13), and (A15) we have

$$
\begin{aligned}
& \boldsymbol{\nabla} \times \frac{1}{\mu}(\boldsymbol{\nabla} \times \widetilde{\mathbf{A}})+\frac{\partial^{2}}{\partial t^{2}} \varepsilon \widetilde{\mathbf{A}} \\
&= \boldsymbol{\nabla} \times \frac{1}{\mu}(\boldsymbol{\nabla} \times \mathbf{A})+\boldsymbol{\nabla} \times \frac{1}{\mu}\left(\boldsymbol{\nabla} \times \frac{1}{\varepsilon}(\boldsymbol{\nabla} \times \mathbf{F})\right) \\
&+\frac{\partial^{2}}{\partial t^{2}} \varepsilon A+\frac{\partial^{2}}{\partial t^{2}} \boldsymbol{\nabla} \times \mathbf{F} \\
&= j_{L}-\frac{\partial}{\partial t} \varepsilon \boldsymbol{\nabla} \phi+0+\frac{\partial^{2}}{\partial t^{2}} \boldsymbol{\nabla} \times \mathbf{F} \\
&= \mathbf{j}_{L}-\frac{\partial}{\partial t}(\varepsilon \nabla \widetilde{\phi}) .
\end{aligned}
$$

However, it is not difficult to show that $F$ is, in fact, zero. Since $\boldsymbol{\nabla} \cdot \varepsilon \mathbf{A}, \boldsymbol{\nabla} \cdot \varepsilon \widetilde{\mathbf{A}}, \boldsymbol{\nabla} \cdot(\boldsymbol{\nabla} \times \mathbf{F})$ are all zero it follows that $\mathbf{A}, \widetilde{\mathbf{A}}$, and $(1 / \varepsilon) \boldsymbol{\nabla} \times \mathbf{F}$ can all be expanded in terms of the mode functions $\mathbf{A}_{k}(\mathbf{R})$ [see Eq. (49)],

$$
\begin{gathered}
\mathbf{A}=\sum_{k} q_{k} \mathbf{A}_{k}, \\
\widetilde{A}=\sum_{k} \widetilde{q_{k}} \mathbf{A}_{k}, \\
\frac{1}{\varepsilon} \nabla \times \mathbf{F}=\sum_{k} C_{k} \mathbf{A}_{k} .
\end{gathered}
$$

The coefficients $C_{k}$ are fixed by $\mathbf{F}$. Hence

$$
\widetilde{q_{k}}=q_{k}+C_{k} .
$$

But we have from (A13) and (A21)

$$
\sum_{k} C_{k} \boldsymbol{\nabla} \times \mathbf{A}_{k}=\mathbf{0}
$$

Hence

$$
\sum_{k} C_{k} \boldsymbol{\nabla} \times \frac{1}{\mu}\left(\boldsymbol{\nabla} \times \mathbf{A}_{k}\right)=\mathbf{0} .
$$

For variable $\mu(\mathbf{R})$ the generalized Helmholtz equations (42)

$$
\boldsymbol{\nabla} \times \frac{1}{\mu}\left(\boldsymbol{\nabla} \times \mathbf{A}_{k}\right)=\varepsilon \omega_{k}^{2} \mathbf{A}_{k}
$$

give

$$
\sum_{k} C_{k} \varepsilon \omega_{k}^{2} \mathbf{A}_{k}=0
$$

Multiplying by $A_{k^{\prime}}^{*}$ and integrating gives [using Eq. (45)].

$$
C_{k} \omega_{k}^{2}=0
$$

so that all $C_{k}$ are zero. Hence

$$
\begin{gathered}
\mathbf{F}=\mathbf{0}, \\
\widetilde{\mathbf{A}}=\mathbf{A}, \\
\varepsilon \nabla \widetilde{\phi}=\varepsilon \nabla \phi .
\end{gathered}
$$

Thus $\mathbf{A}$ and $\varepsilon \nabla \phi$ are unique.

\section{APPENDIX B: DERIVATION OF HAMILTONIAN (36)}

The Hamiltonian density is

$$
\begin{aligned}
\mathcal{H}_{C}^{\prime} & =\boldsymbol{\pi} \cdot \frac{\partial \mathbf{A}}{\partial t}-\mathcal{L}_{C}^{\prime} \\
& =\frac{\left(\boldsymbol{\pi}+\mathbf{P}_{L}^{\prime}\right)^{2}}{2 \varepsilon}+\frac{(\boldsymbol{\nabla} \times \mathbf{A})^{2}}{2 \mu}-\mathbf{M}_{L} \cdot \boldsymbol{\nabla} \times \mathbf{A}
\end{aligned}
$$

on substituting for $\dot{\mathbf{A}}$ from (35). The Hamiltonian is

$$
H^{\prime}=\sum_{\xi \alpha} \mathbf{p}_{\xi \alpha} \cdot \dot{\mathbf{r}}_{\xi \alpha}-\sum_{\xi \alpha} \frac{1}{2} M_{\xi \alpha} \dot{\mathbf{r}}_{\xi \alpha}^{2}+V_{\mathrm{Coul}}+\int d^{3} \mathbf{R} \mathcal{H}_{C}^{\prime},
$$

where we substitute for the particle velocities $\dot{\mathbf{r}}_{\xi \alpha}$ from (34), including inside the term $\int d^{3} \mathbf{R} \mathbf{M}_{\mathrm{L}} \cdot \boldsymbol{\nabla} \times \mathbf{A}$, and where $\mathbf{M}_{L}$ is given by (32). For the latter term 


$$
\int d^{3} \mathbf{R} \mathbf{M}_{L} \cdot(\boldsymbol{\nabla} \times \mathbf{A})=\sum_{\xi \alpha} \dot{\mathbf{r}}_{\xi \alpha} \cdot \int_{0}^{1} u d u q_{\xi \alpha} \mathbf{B}\left(\mathbf{R}_{\xi}+u\left(\mathbf{r}_{\xi \alpha}-\mathbf{R}_{\xi}\right)\right) \times\left(\mathbf{r}_{\xi \alpha}-\mathbf{R}_{\xi}\right)
$$

so that after some vector algebra we find that

$$
H^{\prime}=\sum_{\xi \alpha} \frac{\left[\mathbf{p}_{\xi \alpha}-\int_{0}^{1} u d u q_{\xi \alpha} \mathbf{B}\left(\mathbf{R}_{\xi}+u\left(\mathbf{r}_{\xi \alpha}-\mathbf{R}_{\xi}\right)\right) \times\left(\mathbf{r}_{\xi \alpha}-\mathbf{R}_{\xi}\right)\right]^{2}}{2 M_{\xi \alpha}}+V_{\text {Coul }}+\int d^{3} \mathbf{R}\left(\frac{\left(\boldsymbol{\pi}+\mathbf{P}_{L}^{\prime}\right)^{2}}{2 \varepsilon}+\frac{(\boldsymbol{\nabla} \times \mathbf{A})^{2}}{2 \mu}\right)
$$

With the reduced magnetization density given by (37) we see that the cross term in (B5) obtained by expanding the first term is

$$
\begin{aligned}
\int d^{3} \mathbf{R} \mathbf{M}_{L}^{\prime}(\mathbf{R}) \cdot \mathbf{B}(\mathbf{R}) & =\sum_{\xi \alpha} q_{\xi \alpha} \int_{0}^{1} u d u\left(\mathbf{r}_{\xi \alpha}-\mathbf{R}_{\xi}\right) \times \frac{\mathbf{p}_{\xi \alpha}}{M_{\xi \alpha}} \cdot \mathbf{B}\left(\mathbf{R}_{\xi}+u\left(\mathbf{r}_{\xi \alpha}-\mathbf{R}_{\xi}\right)\right) \\
& =\sum_{\xi \alpha} \frac{\mathbf{p}_{\xi \alpha}}{M_{\xi \alpha}} \cdot \int_{0}^{1} u d u q_{\xi \alpha} \mathbf{B}\left(\mathbf{R}_{\xi}+u\left(\mathbf{r}_{\xi \alpha}-\mathbf{R}_{\xi}\right)\right) \times\left(\mathbf{r}_{\xi \alpha}-\mathbf{R}_{\xi}\right) .
\end{aligned}
$$

Substituting (B6) into (B5) the new Hamiltonian (36) then follows.

\section{APPENDIX C: PROPERTIES OF VECTOR MODE FUNCTIONS}

The harmonic solutions $\mathbf{A}_{k}(\mathbf{R})$ of the generalized inhomogeneous wave equations (21) for zero source terms satisfies the generalized Helmholtz equation (42), which can be written as an eigenvalue equation in terms of an operator $L$,

$$
L \mathbf{A}_{k}=\lambda_{k} \mathbf{A}_{k}
$$

where

$$
L=\frac{1}{\varepsilon}\left(\nabla \times \frac{1}{\mu}(\nabla \times \cdots)\right)
$$

and the eigenvalues $\lambda_{k}$ equal $\omega_{k}^{2}$. The differential operator $L$ is self-adjoint in that for arbitrary vector fields $\mathbf{U}, \mathbf{V}$ (which are periodic over the space of integration)

$$
\int d^{3} \mathbf{R} \varepsilon(\mathbf{R}) \mathbf{U}^{*} \cdot(L \mathbf{V})=\int d^{3} \mathbf{R} \varepsilon(\mathbf{R})(L \mathbf{U})^{*} \cdot \mathbf{V}
$$

Here the electric permittivity function $\varepsilon(\mathbf{R})$ acts as a positive weight function. To show this result we note that the left and right sides can be written as

$$
\begin{aligned}
& I_{L}=\int d^{3} \mathbf{R} \mathbf{U}^{*} \cdot\left(\boldsymbol{\nabla} \times \frac{1}{\mu}(\boldsymbol{\nabla} \times \mathbf{V})\right), \\
& I_{R}=\int d^{3} \mathbf{R}\left(\boldsymbol{\nabla} \times \frac{1}{\mu}\left(\boldsymbol{\nabla} \times \mathbf{U}^{*}\right)\right) \cdot \mathbf{V} .
\end{aligned}
$$

Now

$$
\begin{aligned}
\boldsymbol{\nabla} \cdot\left(\mathbf{U}^{*} \times \frac{1}{\mu}(\boldsymbol{\nabla} \times \mathbf{V})\right)= & \frac{1}{\mu}(\boldsymbol{\nabla} \times \mathbf{V}) \cdot\left(\boldsymbol{\nabla} \times \mathbf{U}^{*}\right) \\
& -\mathbf{U}^{*} \cdot \boldsymbol{\nabla} \times\left(\frac{1}{\mu}(\boldsymbol{\nabla} \times \mathbf{V})\right), \\
\boldsymbol{\nabla} \cdot\left(\mathbf{V} \times \frac{1}{\mu}\left(\boldsymbol{\nabla} \times \mathbf{U}^{*}\right)\right)= & \frac{1}{\mu}\left(\boldsymbol{\nabla} \times \mathbf{U}^{*}\right) \cdot(\boldsymbol{\nabla} \times \mathbf{V}) \\
& -\mathbf{V} \cdot \boldsymbol{\nabla} \times \frac{1}{\mu}\left(\boldsymbol{\nabla} \times \mathbf{U}^{*}\right) .
\end{aligned}
$$

Integrating each side of the equations (C5), and noting that the integrals of the divergence terms are zero, we find that

$$
\begin{aligned}
& I_{L}=\int d^{3} \mathbf{R} \frac{1}{\mu}(\boldsymbol{\nabla} \times \mathbf{V}) \cdot\left(\boldsymbol{\nabla} \times \mathbf{U}^{*}\right), \\
& I_{R}=\int d^{3} \mathbf{R} \frac{1}{\mu}\left(\boldsymbol{\nabla} \times \mathbf{U}^{*}\right) \cdot(\boldsymbol{\nabla} \times \mathbf{V}) .
\end{aligned}
$$

As these expressions are the same the result (C3) follows. The properties of the $\mathbf{A}_{k}$ and the $\omega_{k}$ then follow from similar proofs to those used in quantum theory.

To show that the eigenvalues $\lambda_{k}$ are real, each side of Eq. (C1) is multiplied by $\varepsilon(R) \mathbf{A}_{k}^{*}$ in a scalar product and then integrated over $\mathbf{R}$. The use of (C3) gives

$$
\begin{aligned}
\int d^{3} \mathbf{R} \varepsilon(\mathbf{R}) \mathbf{A}_{k}^{*} \cdot\left(L \mathbf{A}_{k}\right) & =\lambda_{k} \int d^{3} \mathbf{R} \varepsilon(\mathbf{R}) \mathbf{A}_{k}^{*} \cdot \mathbf{A}_{k} \\
& =\int d^{3} \mathbf{R} \varepsilon(\mathbf{R})\left(L \mathbf{A}_{k}\right)^{*} \cdot \mathbf{A}_{k}
\end{aligned}
$$

The right-hand side of (C7) is the complex conjugate of the left-hand side showing both to be real. As the quantity 
$\varepsilon(\mathbf{R}) \mathbf{A}_{k}^{*} \cdot \mathbf{A}_{k}$ is real, the reality of $\lambda_{k}$ follows. The result that $\lambda_{k}$ is positive, and hence equal to the square of a positive real frequency $\omega_{k}$, follows via the use of the form (C6) for the left-hand side of (C7). We see that

$$
\int d^{3} \mathbf{R} \frac{1}{\mu}\left(\boldsymbol{\nabla} \times \mathbf{A}_{k}\right) \cdot\left(\boldsymbol{\nabla} \times \mathbf{A}_{k}^{*}\right)=\lambda_{k} \int d^{3} \mathbf{R} \varepsilon(\mathbf{R}) \mathbf{A}_{k}^{*} \cdot \mathbf{A}_{k} .
$$

As the integrals are clearly positive, then so must be $\lambda_{k}$.

To show that the mode functions $\mathbf{A}_{k}$ are orthogonal as in Eq. (45) for different $\omega_{k}$, we apply (C1) for a different eigenvalue $\lambda_{l}$, multiply by $\varepsilon(\mathbf{R}) \mathbf{A}_{k}^{*}$ in a scalar product, and integrate over $\mathbf{R}$. Thus gives

$$
\int d^{3} \mathbf{R} \varepsilon(\mathbf{R}) \mathbf{A}_{k}^{*} \cdot\left(L \mathbf{A}_{l}\right)=\lambda_{l} \int d^{3} \mathbf{R} \varepsilon(\mathbf{R}) \mathbf{A}_{k}^{*} \cdot \mathbf{A}_{l}
$$

Reverting to $(\mathrm{C} 1)$ and multiplying by $\varepsilon(\mathbf{R}) \mathbf{A}_{l}^{*}$ in a scalar product and integrating gives

$$
\int d^{3} \mathbf{R} \varepsilon(\mathbf{R}) \mathbf{A}_{l}^{*} \cdot\left(L \mathbf{A}_{k}\right)=\lambda_{k} \int d^{3} \mathbf{R} \varepsilon(\mathbf{R}) \mathbf{A}_{l}^{*} \cdot \mathbf{A}_{k} .
$$

Taking the complex conjugate of each side of (C10), using $\lambda_{k}$ real and the adjoint condition (C3) gives

$$
\int d^{3} \mathbf{R} \varepsilon(\mathbf{R}) \mathbf{A}_{k}^{*} \cdot\left(L \mathbf{A}_{l}\right)=\lambda_{k} \int d^{3} \mathbf{R} \varepsilon(\mathbf{R}) \mathbf{A}_{k}^{*} \cdot \mathbf{A}_{l} .
$$

Since the left sides of (C9) and (C11) are equal it follows that if $\lambda_{k} \neq \lambda_{l}$ the orthogonality condition (C12) applies.

$$
\int d^{3} \mathbf{R} \varepsilon(\mathbf{R}) \mathbf{A}_{k}^{*} \cdot \mathbf{A}_{l}=0 .
$$

\section{APPENDIX D: DERIVATION OF LAGRANGIAN (56)}

The various terms in Eq. (33) are evaluated via substituting for the vector potential A via Eq. (49) or its complex conjugate form, and introducing the half-space expressions after simplification. We have

$$
\begin{aligned}
\int d^{3} \mathbf{R} \frac{1}{2} \varepsilon\left(\frac{\partial \mathbf{A}}{\partial t}\right)^{2} & =\frac{1}{2} \sum_{k l} \dot{q}_{k}^{*} \dot{q}_{l} \int d^{3} \mathbf{R} \varepsilon(\mathbf{R}) \mathbf{A}_{k}^{*}(\mathbf{R}) \cdot \mathbf{A}_{l}(\mathbf{R}) \\
& =\frac{1}{2} \sum_{k l} \dot{q}_{k}^{*} \dot{q}_{l} \delta_{k l}
\end{aligned}
$$$$
\text { [using orthogonality (45)] }
$$$$
=\frac{1}{2} \sum_{k} \dot{q}_{k}^{*} \dot{q}_{k}=\sum_{k}{ }^{\prime} \dot{q}_{k}^{*} \dot{q}_{k}
$$

using the relationship (51) between the generalized coordinates of the $k$ and $k^{*}$ modes. Also

$$
\begin{aligned}
\int d^{3} \mathbf{R} \frac{1}{2 \mu}(\boldsymbol{\nabla} \times \mathbf{A})^{2} \\
=\sum_{k l} q_{k}^{*} q_{l} \int d^{3} \mathbf{R} \frac{1}{2 \mu}\left(\boldsymbol{\nabla} \times \mathbf{A}_{k}^{*}\right) \cdot\left(\boldsymbol{\nabla} \times \mathbf{A}_{l}\right) .
\end{aligned}
$$

Using

$$
\begin{aligned}
\boldsymbol{\nabla} \cdot\left(\mathbf{A}_{l} \times \frac{1}{\mu}\left(\boldsymbol{\nabla} \times \mathbf{A}_{k}^{*}\right)\right)= & \frac{1}{\mu}\left(\boldsymbol{\nabla} \times \mathbf{A}_{k}^{*}\right) \cdot\left(\boldsymbol{\nabla} \times \mathbf{A}_{l}\right) \\
& -\mathbf{A}_{l} \cdot\left(\boldsymbol{\nabla} \times \frac{1}{\mu}\left(\boldsymbol{\nabla} \times \mathbf{A}_{k}^{*}\right)\right)
\end{aligned}
$$

and with the integral of the divergence term giving zero we find that

$$
\begin{aligned}
\int & d^{3} \mathbf{R} \frac{1}{2 \mu}(\boldsymbol{\nabla} \times \mathbf{A})^{2} \\
= & \frac{1}{2} \sum_{k l} q_{k}^{*} q_{l} \int d^{3} \mathbf{R}\left(\boldsymbol{\nabla} \times \frac{1}{\mu}\left(\boldsymbol{\nabla} \times \mathbf{A}_{k}^{*}\right)\right) \cdot \mathbf{A}_{l} \\
= & \frac{1}{2} \sum_{k l} q_{k}^{*} q_{l} \int d^{3} \mathbf{R} \omega_{k}^{2} \varepsilon(\mathbf{R}) \mathbf{A}_{k}^{*} \cdot \mathbf{A}_{l} \\
= & \frac{1}{2} \sum_{k} \omega_{k}^{2} q_{k}^{*} q_{k} \\
= & \sum_{k}{ }^{\prime} \omega_{k}^{2} q_{k}^{*} q_{k}
\end{aligned}
$$

using the generalized Helmholtz equation (42), and orthogonality conditions (45) and (51) again. Finally

$$
\begin{aligned}
& \int d^{3} \mathbf{R} \mathbf{P}_{L}^{\prime} \cdot\left(\frac{\partial \mathbf{A}}{\partial t}\right) \\
& =\sum_{k} \dot{q}_{k}^{*} \int d^{3} \mathbf{R} \mathbf{P}_{L}^{\prime} \cdot \mathbf{A}_{k}^{*} \\
& =\sum_{k l} \dot{q}_{k}^{*} \int d^{3} \mathbf{R}^{\prime} \mathbf{P}_{L} \cdot \mathbf{A}_{l}^{*} \int d^{3} \mathbf{R} \varepsilon(\mathbf{R}) \mathbf{A}_{k}^{*} \cdot \mathbf{A}_{l} \\
& =\sum_{k} \dot{q}_{k}^{*} \int d^{3} \mathbf{R} \mathbf{P}_{L} \cdot \mathbf{A}_{k}^{*} \\
& =\sum_{k}\left(\dot{q}_{k}^{*} \int d^{3} \mathbf{R} \mathbf{P}_{L} \cdot \mathbf{A}_{k}^{*}+\dot{q}_{k} \int d^{3} \mathbf{R} \mathbf{P}_{L} \cdot \mathbf{A}_{k}\right)
\end{aligned}
$$

using (53) and orthogonality (45).

\section{APPENDIX E: DERIVATION OF MULTIPOLAR HAMILTONIAN (61)}

Our Hamiltonian is given by

$$
H^{\prime}=\sum_{\xi \alpha} \mathbf{p}_{\xi \alpha} \cdot \dot{\mathbf{r}}_{\xi \alpha}+\sum_{k}^{\prime}\left(p_{k} \dot{q}_{k}^{*}+p_{k}^{*} \dot{q}_{k}\right)-L^{\prime},
$$


where $\mathbf{r}_{\xi \alpha}, \dot{q}_{k}^{*}, \dot{q}_{k^{*}}^{*},=\dot{q}_{k}$ are replaced by their expressions in terms of the generalized momenta (34) and (57). Then with the Lagrangian as in (56)

$$
\begin{aligned}
H^{\prime}= & \sum_{\xi \alpha} \mathbf{p}_{\xi \alpha} \cdot\left(\frac{\mathbf{p}_{\xi \alpha}-\int_{0}^{1} u d u q_{\xi \alpha} \mathbf{B}\left(\mathbf{R}_{\xi}+u\left(\mathbf{r}_{\xi \alpha}-\mathbf{R}_{\xi}\right)\right) \times\left(\mathbf{r}_{\xi \alpha}-\mathbf{R}_{\xi}\right)}{M_{\xi \alpha}}\right) \\
& -\sum_{\xi \alpha} \frac{1}{2} M_{\xi \alpha}\left(\frac{\mathbf{p}_{\xi \alpha}-\int_{0}^{1} u d u q_{\xi \alpha} \mathbf{B}\left(\mathbf{R}_{\xi}+u\left(\mathbf{r}_{\xi \alpha}-\mathbf{R}_{\xi}\right)\right) \times\left(\mathbf{r}_{\xi \alpha}-\mathbf{R}_{\xi}\right)}{M_{\xi \alpha}}\right)+V_{\text {Coul }} \\
& -\sum_{\xi \alpha}\left(\frac{\mathbf{p}_{\xi \alpha}-\int_{0}^{1} u d u q_{\xi \alpha} \mathbf{B}\left(\mathbf{R}_{\xi}+u\left(\mathbf{r}_{\xi \alpha}-\mathbf{R}_{\xi}\right)\right) \times\left(\mathbf{r}_{\xi \alpha}-\mathbf{R}_{\xi}\right)}{M_{\xi \alpha}}\right) \cdot \int_{0}^{1} u d u q_{\xi \alpha} \mathbf{B}\left(R_{\xi}+u\left(\mathbf{r}_{\xi \alpha}-\mathbf{R}_{\xi}\right)\right) \times\left(\mathbf{r}_{\xi \alpha}-\mathbf{R}_{\xi}\right) \\
& +\sum_{k}^{\prime}\left[p_{k}\left(p_{k}^{*}+\int d^{3} \mathbf{R} \mathbf{P}_{L} \cdot \mathbf{A}_{k}\right)+p_{k}^{*}\left(p_{k}+\int d^{3} \mathbf{R} \mathbf{P}_{L} \cdot \mathbf{A}_{k}^{*}\right)\right]-\sum_{k}^{\prime}\left(p_{k}+\int d^{3} \mathbf{R} \mathbf{P}_{L} \cdot \mathbf{A}_{k}^{*}\right)\left(p_{k}^{*}+\int d^{3} \mathbf{R} \mathbf{P}_{L} \cdot \mathbf{A}_{k}\right) \\
& +\sum_{k}^{\prime} \omega_{k}^{2} q_{k} q_{k}^{*}+\sum_{k}^{\prime}\left\{\left(p_{k}+\int d^{3} \mathbf{R} \mathbf{P}_{L} \cdot \mathbf{A}_{k}^{*}\right) \int d^{3} \mathbf{R} \mathbf{P}_{L} \cdot \mathbf{A}_{k}+\left(p_{k}^{*}+\int d^{3} \mathbf{R} \mathbf{P}_{L} \cdot \mathbf{A}_{k}\right) \int d^{3} \mathbf{R} \mathbf{P}_{L} \cdot \mathbf{A}_{k}^{*}\right\} .
\end{aligned}
$$

Hence

$$
\begin{aligned}
H^{\prime}= & \sum_{\xi \alpha} \frac{\left(\mathbf{p}_{\xi \alpha}-\int_{0}^{1} u d u q_{\xi \alpha} \mathbf{B}\left(\mathbf{R}_{\xi}+u\left(\mathbf{r}_{\xi \alpha}-\mathbf{R}_{\xi}\right)\right) \times\left(\mathbf{r}_{\xi \alpha}-\mathbf{R}_{\xi}\right)\right)^{2}}{2 M_{\xi \alpha}}+V_{\mathrm{Coul}}+\sum_{k}{ }^{\prime}\left(\int d^{3} \mathbf{R} \mathbf{P}_{L} \cdot \mathbf{A}_{k}^{*}\right)\left(\int d^{3} R \mathbf{P}_{L} \cdot \mathbf{A}_{k}\right) \\
& +\sum_{k}^{\prime}\left(p_{k} q_{k}^{*}+\omega_{k}^{2} q_{k} q_{k}^{*}\right)+\sum_{k}^{\prime}\left(p_{k} \int d^{3} R \mathbf{P}_{L} \cdot \mathbf{A}_{k}+p_{k}^{*} \int d^{3} R \mathbf{P}_{L} \cdot \mathbf{A}_{k}^{*}\right) .
\end{aligned}
$$

The cross terms associated with the expansion of the first term is treated as in Appendix B to give

$$
\begin{aligned}
\sum_{\xi \alpha} & \mathbf{p}_{\xi \alpha} \cdot \int_{0}^{1} u d u \frac{q_{\xi \alpha}}{M_{\xi \alpha}} \mathbf{B}\left(\mathbf{R}_{\xi}+u\left(\mathbf{r}_{\xi \alpha}-\mathbf{R}_{\xi}\right)\right) \times\left(\mathbf{r}_{\xi \alpha}-\mathbf{R}_{\xi}\right) \\
= & \int d^{3} \mathbf{R} \mathbf{M}_{L}^{\prime} \cdot \mathbf{B}=\sum_{k}{ }^{\prime}\left(q_{k} \int d^{3} R \mathbf{M}_{L}^{\prime} \cdot \boldsymbol{\nabla} \times \mathbf{A}_{k}\right. \\
& \left.+q_{k}^{*} \int d^{3} \mathbf{R} \mathbf{M}_{L}^{\prime} \cdot \boldsymbol{\nabla} \times \mathbf{A}_{k}^{*}\right) .
\end{aligned}
$$

The particle kinetic energy and the diamagnetic energy term arise from the other contributions from the expansion of the first term in (E3). Combining (E3) and (E4) the multipolar Hamiltonian (61) is obtained.

\section{APPENDIX F: RADIATION FIELD ENERGY TERM}

We start from the form given in the multipolar Hamiltonian (61),

$$
H_{F}=\sum_{k} \frac{1}{2}\left(p_{k} p_{k}^{*}+\omega_{k}^{2} q_{k} q_{k}^{*}\right)
$$

This becomes, on replacing the $q_{k}, p_{k}$ by quantum operators,

$$
\begin{aligned}
\hat{H}_{F}= & \sum_{k} \frac{1}{2}\left(\hat{p}_{k} \hat{p}_{k}^{\dagger}+\omega_{k}^{2} \hat{q}_{k} \hat{q}_{k}^{\dagger}\right) \\
= & \sum_{k}\left\{\frac{1}{2}\left(\frac{1}{i} \sqrt{\frac{\hbar \omega_{k}}{2}}\left(\hat{a}_{k}-\hat{a}_{k^{*}}^{\dagger}\right)\right)\right. \\
& \times\left(-\frac{1}{i} \sqrt{\frac{\hbar \omega_{k}}{2}}\left(\hat{a}_{k}^{\dagger}-\hat{a}_{k^{*}}\right)\right) \\
& \left.+\frac{1}{2} \omega_{k}^{2} \sqrt{\frac{\hbar}{2 \omega_{k}}} \sqrt{\frac{\hbar}{2 \omega_{k}}}\left(\hat{a}_{k}+\hat{a}_{k^{*}}^{\dagger}\right)\left(\hat{a}_{k}^{\dagger}+\hat{a}_{k^{*}}\right)\right\} \\
= & \sum_{k} \frac{1}{4} \hbar \omega_{k}\left\{\hat{a}_{k} \hat{a}_{k}^{\dagger}-\hat{a}_{k} \hat{a}_{k^{*}}-\hat{a}_{k^{*}}^{\dagger} \hat{a}_{k}^{\dagger}+\hat{a}_{k^{*}}^{\dagger} \hat{a}_{k^{*}}+\hat{a}_{k} \hat{a}_{k}^{\dagger}\right. \\
& \left.+\hat{a}_{k} \hat{a}_{k^{*}}+\hat{a}_{k^{*}}^{\dagger} \hat{a}_{k}^{\dagger}+\hat{a}_{k^{*}}^{\dagger} \hat{a}_{k^{*}}\right\} \\
= & \sum_{k} \frac{1}{2} \hbar \omega_{k}\left(\hat{a}_{k} \hat{a}_{k}^{\dagger}+\hat{a}_{k^{*}}^{\dagger} \hat{a}_{k^{*}}\right) \\
= & \sum_{k} \frac{1}{2} \hbar \omega_{k}\left(\hat{a}_{k} \hat{a}_{k}^{\dagger}+\hat{a}_{k}^{\dagger} \hat{a}_{k}\right) .
\end{aligned}
$$

Using the commutation rules (70) we find that 


$$
\hat{H}_{F}=\sum_{k}\left(\hat{a}_{k}^{\dagger} \hat{a}_{k}+\frac{1}{2}\right) \hbar \omega_{k},
$$

the same as that for a set of quantum harmonic oscillators.

\section{APPENDIX G: TRANSVERSE AND LONGITUDINAL COMPONENTS OF $P_{L}$ IN THE ELECTRIC DIPOLE APPROXIMATION}

The transverse $\mathbf{F}_{\perp}(\mathbf{R})$ and longitudinal $\mathbf{F}_{\|}(\mathbf{R})$ components of a vector field $\mathbf{F}(\mathbf{R})$ may be calculated via Helmholtz theorem [10] via

$$
\begin{gathered}
\mathbf{F}(\mathbf{R})=\mathbf{F}_{\|}(\mathbf{R})+\mathbf{F}_{1}(\mathbf{R}), \\
\mathbf{F}_{\|}(\mathbf{R})=\nabla \phi, \\
\mathbf{F}_{\perp}(\mathbf{R})=\nabla \times \mathbf{A},
\end{gathered}
$$

where $\phi, \mathbf{A}$ are given by

$$
\begin{gathered}
\phi=-\boldsymbol{\nabla} \cdot \mathbf{X}, \\
\mathbf{A}=\boldsymbol{\nabla} \times \mathbf{X},
\end{gathered}
$$

with the field $\mathbf{X}(\mathbf{R})$ obtained from $\mathbf{F}(\mathbf{R})$ via

$$
\mathbf{X}(\mathbf{R})=\frac{1}{4 \pi} \int d^{3} \mathbf{R}^{\prime} \frac{\mathbf{F}\left(\mathbf{R}^{\prime}\right)}{\left|\mathbf{R}-\mathbf{R}^{\prime}\right|}
$$

In this case

$$
\boldsymbol{\nabla} \cdot \mathbf{A}=0
$$

The polarization density in the electric dipole approximation is

$$
\mathbf{P}_{L}(\mathbf{R})=\sum_{\xi} \boldsymbol{\mu}_{\xi} \delta\left(\mathbf{R}-\mathbf{R}_{\xi}\right)
$$

A straightforward evaluation gives

$$
\begin{gathered}
\mathbf{X}(\mathbf{R})=\frac{1}{4 \pi} \sum_{\xi} \frac{\boldsymbol{\mu}_{\xi}}{\left|\mathbf{R}-\mathbf{R}_{\xi}\right|}, \\
\phi=\frac{1}{4 \pi} \sum_{\xi} \frac{\left(\mathbf{R}-\mathbf{R}_{\xi}\right) \cdot \boldsymbol{\mu}_{\xi}}{\left|\mathbf{R}-\mathbf{R}_{\xi}\right|^{3}}, \\
\mathbf{A}=-\frac{1}{4 \pi} \sum_{\xi} \frac{\left(\mathbf{R}-\mathbf{R}_{\xi}\right) \times \boldsymbol{\mu}_{\xi}}{\left|\mathbf{R}-\mathbf{R}_{\xi}\right|^{3}}, \\
\left.\mathbf{R} \neq \mathbf{P}_{L}\right)_{\|}, \quad \frac{3\left(\mathbf{R}-\mathbf{R}_{\xi}\right) \cdot \boldsymbol{\mu}_{\xi}\left(\mathbf{R}-\mathbf{R}_{\xi}\right)}{4 \pi} \sum_{\xi}\left(\frac{\boldsymbol{\mu}_{\xi}-\left.\mathbf{R}_{\xi}\right|^{5}}{\left|\mathbf{R}-\mathbf{R}_{\xi}\right|^{3}}-\frac{(\mathrm{G}}{(\mathrm{G}}\right), \\
\left(\mathbf{P}_{L}\right)_{\perp}=-\left(\mathbf{P}_{L}\right)_{\|}
\end{gathered}
$$

\section{APPENDIX H: MODE FUNCTIONS FOR ONE-DIMENSIONAL CAVITY}

The mode functions $\mathbf{A}_{k}(\mathbf{R})$ for the one-dimensional Fabry-Perot cavity shown in Fig. 2 will be assumed to have polarization vector along the $x$ axis and the mode functions will only depend on the $z$ coordinate,

$$
\mathbf{A}_{k}(\mathbf{R})=\mathbf{i} A_{k}(z)
$$

From the generalized Helmholtz equation (42) we obtain

$$
\frac{d^{2} A_{k}}{d z^{2}}+\omega_{k}^{2} \mu_{0} \varepsilon(z) A_{k}=0,
$$

where $\varepsilon(z)=\varepsilon_{0}$ everywhere except for $-d \leqslant z \leqslant 0$, where $\varepsilon(z)=\kappa_{1} \varepsilon_{0} \cdot \mu=\mu_{0}$ everywhere.

The continuity of the tangential components of $\mathbf{E}$ and $\mathbf{H}$ show that $A_{k}(z)$ and $d A_{k} / d z$ are continuous at $z=-d$ and $z=0$ and we take as boundary conditions

$$
A_{k}(l)=0 \text {, }
$$

$$
A_{k}(-L-d)=0 \text {, }
$$

corresponding to the mode functions terminating at the perfect mirrors.

From the Helmholtz equation (H2) the mode functions are essentially sine functions in the cavity, dielectric, and external regimes, respectively:

$A_{k}(z)=\left\{\begin{array}{l}\alpha_{1} \sin k(z-l), \quad l \geqslant z \geqslant 0 \\ \beta_{1} \sin \sqrt{\kappa_{1}} k\left(z+z_{2}\right), \quad 0 \geqslant z \geqslant-d \\ \gamma_{1} \sin k(z+L+d), \quad-d \geqslant z \geqslant-(L+d) .\end{array}\right.$

In these equations $k=\omega_{k} / c$ is the wave number, $\alpha_{1}, \beta_{1}$, and $\gamma_{1}$ are amplitudes, and $z_{2}$ is a shift term.

The thin dielectric approximation is based on the wavelength in the dielectric layer being much longer than its thickness,

$$
\sqrt{\kappa_{1}} k d \ll 1
$$

Associated with the dielectric layer is a strength parameter $\Lambda=\kappa_{1} k d$ which for the strong dielectric approximation is much larger than unity,

$$
\kappa_{1} k d \gg 1 \text {. }
$$

Applying the continuity conditions to the case of the thin, strong dielectric leads to a mode equation for the wave vector $k$,

$$
f(k)=\tan k L=\frac{\tan k l}{\Lambda \tan k l-1}=g(k) .
$$

This is a transcendental equation. The assymptotes for the function on the left hand side $[f(k)]$ are separated by $\pi / L$. As $L \gg l$ the function on the right hand side $[g(k)]$ is a much more slowly varying function of $k$ apart from perhaps near its asymptotes (where $\tan k l=1 / \Lambda$ ). Thus $g(k)$ will cross $f(k)$ once between each of the assymptotes of the latter, giving modes with an average separation $\Delta k \sim \pi / L$. Thus the 
actual or true modes form a quasicontinuum, the separation being given by the universe length $L$.

The Fabry-Perot resonances occur at wave vectors $k_{n}$ (not themselves necessarily equal to a mode wave number $k$ ) given by the assymptotes of $g(k)$ such that

$$
\begin{aligned}
k_{n} & =\frac{n \pi}{l}+\frac{1}{l} \tan ^{-1}\left(\Lambda_{n}^{-1}\right) \\
& \approx \frac{n \pi}{l}+\frac{1}{l \Lambda_{n}},
\end{aligned}
$$

where $n$ is an integer and the strength factor $\Lambda_{n}$ is

$$
\Lambda_{n}=\kappa_{1} k_{n} d .
$$

As can be seen from (H9) the strength factor $\Lambda_{n}$ determines the shift in Fabry-Perot wave number $k_{n}$ from the value $n \pi /$ $l$. This notional wave number $n \pi / l$ corresponds to there being $n$ half wavelengths inside the cavity of length $l$. A further parameter that is important is the width factor $\Gamma_{n}$ given by

$$
\Gamma_{n}=\frac{1}{\Lambda_{n}^{2} l} .
$$

The ratio of the cavity region to outside region amplitudes can also obtained by applying the continuity conditions. For wave numbers $k$ near to a resonance $k_{n}$ the expression for $\left(\alpha_{1} / \gamma_{1}\right)^{2}$ is of Lorentzian form,

$$
\left(\frac{\alpha_{1}}{\gamma_{1}}\right)^{2} \approx \frac{\Lambda_{n}^{2} \Gamma_{n}^{2}}{\left(k-k_{n}\right)^{2}+\Gamma_{n}^{2}} \quad \text { for }\left|k-k_{n}\right| \ll \Gamma_{n} \Lambda_{n} .
$$

Thus it is clear that the square of the strength factor $\Lambda_{n}^{2}$ defines the peak value for $\left(\alpha_{1} / \gamma_{1}\right)^{2}$, which occurs on resonance with $k=k_{n}$. As $\Lambda_{n} \gg 1$ the cavity region amplitude is very large compared to the outside region amplitude for $k$ values near resonance. Furthermore it is seen that $\Gamma_{n}$ does in fact define the half width of the Lorentzian form for $\left(\alpha_{1} / \gamma_{1}\right)^{2}$.

The normalization condition for the mode functions in a volume $V=L \mathcal{A}$ (where $\mathcal{A}$ is a cross-sectional area for the system) is obtained from (45) as

$$
\int d z \varepsilon(z) A_{k}^{2}(z)=1 / \mathcal{A}
$$

Substituting the forms (H5) for the mode functions and making appropriate approximations leads to the result

$$
\frac{1}{\mathcal{A}} \approx \frac{1}{2} \varepsilon_{0} \alpha_{1}^{2} l+\frac{1}{2} \varepsilon_{0} \gamma_{1}^{2} L, \quad \text { for }\left|k-k_{n}\right| \ll \Gamma_{n} \Lambda_{n} .
$$

The average separation $\Delta k \sim \pi / L$ of the wave vectors is assumed to be small in comparison to half width $\Gamma_{n}$ of the Lorentzian (H13) that gives the square of the ratio of cavity region to outside region amplitudes.

$$
\frac{\pi}{L} \ll \Gamma_{n} .
$$

Substituting for $\Gamma_{n}$ from (H12) the previous condition leads to

$$
\frac{l}{L} \ll \frac{1}{\pi \Lambda_{n}^{2}}
$$

Since the largest value of $\left(\alpha_{1} / \gamma_{1}\right)^{2}$ is $\Lambda_{n}^{2}$ (at resonance) it follows that

$$
\alpha_{1}^{2} l \ll \gamma_{1}^{2} L
$$

and hence the first term in the normalization result (H15) may be ignored, giving

$$
\gamma_{1} \approx \sqrt{\frac{2}{\varepsilon_{0} L \mathcal{A}}}
$$

This is the same normalization that would apply for mode functions in a free space with volume $V=L \mathcal{A}$. The expression for the cavity region amplitude then becomes

$$
\alpha_{1} \approx \sqrt{\frac{2}{\varepsilon_{0} L \mathcal{A}}} \frac{\Lambda_{n} \Gamma_{n}}{\left[\left(k-k_{n}\right)^{2}+\Gamma_{n}^{2}\right]^{1 / 2}} .
$$

Thus the mode function inside the cavity is given by

$$
\begin{aligned}
& A_{k}(z)= \sqrt{\frac{2}{\varepsilon_{0} L \mathcal{A}}} \frac{\Lambda_{n} \Gamma_{n}}{\left[\left(k-k_{n}\right)^{2}+\Gamma_{m}\right]^{1 / 2}} \sin k(z-l), \\
& l \geqslant z \geqslant 0,
\end{aligned}
$$

assuming that $\left|k-k_{n}\right| \ll \Lambda_{n} \Gamma_{n}$, and so this result is certainly valid for $\left|k-k_{n}\right| \sim \Gamma_{n}$, corresponding to the regime of wave numbers close to the Fabry-Perot resonance.
[1] C. Cohen-Tannoudji, J. Dupont-Roc, and G. Grynberg, Photons and Atoms (Wiley, New York, 1989).

[2] B. Huttner and S. M. Barnett, Phys. Rev. A 46, 4306 (1992); S. T. Ho and P. Kumar, J. Opt. Soc. B 10, 1620 (1993).

[3] E. A. Power, Introductory Quantum Electrodynamics (Longman, London, 1964).

[4] R. J. Glauber and M. Lewenstein, Phys. Rev. A 43, 467 (1991).

[5] L. Knoll, W. Vogel, and D. G. Welsch, Phys. Rev. A 36, 3803 (1987); W. Vogel and D. G. Welsch, Lectures on Quantum
Optics (Akademie Verlag, Berlin, 1994), Chap. 2.

[6] J. C. Penaforte and B. Baseia, Phys. Rev. A 30, 1401 (1984).

[7] J. M. Jauch and K. M. Watson, Phys. Rev. 74, 950 (1948); C. K. Carniglia and L. Mandel, Phys. Rev. D 3, 280 (1971); I. Bialynicki-Birula and J. B. Brogan, ibid. 5, 485 (1972); I. Abram, Phys. Rev. A 35, 4661 (1987).

[8] P. D. Drummond, Phys. Rev. A 42, 6845 (1990); M. Hillery and L. D. Mlodinow, ibid. 30, 1860 (1984).

[9] S. M. Barnett, B. Huttner, and R. Loudon, Phys. Rev. Lett 68, 3698 (1992); G. Bjork, S. Machida, Y. Yamamoto, and K. 
Igeta, Phys. Rev. A 44, 669 (1991).

[10] P. M. Morse and H. Feshbach, Methods of Theoretical Physics (McGraw-Hill, New York, 1953), Vol. 1, p. 53.

[11] E. A. Hinds, Adv. Atom. Mol. Phys. 28, 237 (1991); H. J. Kimble, in Cavity Quantum Electrodynamics, edited by P. R. Berman (Academic Press, New York, 1994), p. 203; S. Haroche, in Fundamental Systems in Quantum Optics, edited by J. Dalibard, J-M. Raimond, and J. Zinn-Justin (North Hol- land, Amsterdam, 1992), p. 767; S. E. Morin, Q. Wu, and T. W. Mossberg, Opt. Photon. News, August (1992), p. 9; E. A. Hinds, in Cavity Quantum Electrodynamics, Ref. [11], p. 1.

[12] R. Lang, M. O. Scully, and W. E. Lamb, Jr., Phys. Rev. A 7, 1788 (1973).

[13] H. M. Lai, P. T. Leung, and K. Young, Phys. Rev. A 37, 1597 (1988).

[14] E. M. Purcell, Phys. Rev. 69, 681 (1946). 